\title{
Scattering Purity and Complexity in Radar Polarimetry
}

\author{
Avik Bhattacharya, Senior Member, IEEE, Subhadip Dey, Graduate Student Member, IEEE, \\ Alejandro C. Frery, Senior Member, IEEE
}

\begin{abstract}
The generalized degree of polarimetric purity is a vital descriptor widely studied and interpreted for electromagnetic wave characterization. It is invariant under the rotation of the reference frame. In this work, we first propose an alternate expression of this purity measure using the mean $(\mathrm{m})$ and standard deviation $(s)$ of the real positive eigenvalues of a Hermitian positive semi-definite matrix. We then use this expression to propose a polarimetric scattering purity and scattering complexity measure. To obtain these expressions, we use certain inequalities on the bounds of the condition number for Hermitian positive definite matrices defined in terms of $m$ and $s$. The polarimetric scattering purity parameter characterizes the overall polarization structure in the scattered wave. In contrast, the polarimetric scattering complexity parameter describes the mixture of orthogonal polarized pure components in the scattered wave. At first, we demonstrate the two proposed measures by analyzing two cases: (i) multiple scattering, and (ii) a mixture of canonical targets. Then, we utilize full-polarimetric $C$ - and L-band Synthetic Aperture Radar (SAR) data to describe the variation of these measures over various land cover classes. We compare their spatial variations over the ocean surface, built-up areas, and vegetation. We observe notable contrasts in the purity and the complexity parameters over a diverse mixture of targets in the scene. Finally, we critically interpret the variation of the two measures over the temporal scene of rice crop acquired by C-band full-polarimetric SAR data. These analyses affirm the importance of these measures for explicit target characterization. The open-source version of the code is available at: https://github.com/Subho07/scattering-purity-and-complexity
\end{abstract}

Index Terms-Polarimetry, degree of polarization, target characterization, scattering purity

\section{INTRODUCTION}

$\mathbf{T}$ ARGET CHARACTERIZATION in radar polarimetry has been a topic of immense interest for decades. The availability of full polarimetric Synthetic Aperture Radar (SAR) data for Earth and Planetary observation has engaged researchers to develop unique techniques to extract relevant scattering descriptors. These descriptors provide valuable scattering information from simple to complex targets.

In this regard, Cloude et al. [1,2] proposed an eigenvectoreigenvalue based analysis of the $3 \times 3$ coherency matrix to quantify target information. They proposed extracting average parameters by introducing a new parameterization of the

A. Bhattacharya and S. Dey are with the Microwave Remote Sensing Lab, Center of Studies in Resources Engineering, Indian Institute of Technology Bombay, India (e-mail: sdey2307@ gmail.com).

Alejandro C. Frery is with the School of Mathematics and Statistics, Victoria University of Wellington, New Zealand, and with the Key Lab of Intelligent Perception and Image Understanding of the Ministry of Education, Xidian University, Xi'an, China (e-mail: alejandro.frery@vuw.ac.nz). eigenvectors, called the $\alpha-\beta$ model, where $\alpha$ is the scatteringtype parameter, and $\beta$ is the target orientation angle.

Utilizing this formalism, they obtained the mean scatteringtype parameter $\bar{\alpha}$ from fully polarimetric SAR data. In order to define the degree of statistical disorder, the polarimetric entropy $H$ (in the von Neumann sense) was introduced as a basisinvariant parameter. These two parameters are widely used in literature for target characterization and classification [3].

Touzi [4] addressed the need to evaluate the robustness of the $\alpha-\beta$ model under a change of wave polarization basis. A new scattering vector model was proposed derived from the con-diagonalization of the Kennaugh-Huynen scattering matrix projected into the Pauli basis. Two parameters, $\left(\alpha_{s}, \Phi_{\alpha_{s}}\right)$ were introduced in the new scattering vector model. Here $\alpha_{s}$ is the angle of the symmetric scattering vector defined in the trihedral-dihedral basis, and $\Phi_{\alpha_{s}}$ is the phase difference between the vector components in the trihedral-dihedral basis. These parameters are widely used in literature for several applications [5-7].

Ratha et al. [8] utilized the geodesic distance (GD) measured over the unit sphere centered at the origin in the space of real $4 \times 4$ matrices. This unit sphere contains all the normalized Kennaugh matrices, equivalent to the second-order information expressed by the $3 \times 3$ coherency (or covariance) matrices. The geodesic distance is suitably used to obtain three rollinvariant parameters: scattering-type $\alpha_{\mathrm{GD}}$, helicity $\tau_{\mathrm{GD}}$, and scattering purity $P_{\mathrm{GD}}$. Furthermore, the authors also proposed a $\left(P_{\mathrm{GD}}, \alpha_{\mathrm{GD}}\right)$ unsupervised classification scheme to cluster full polarimetric SAR images into eight zones.

Unlike the parameterization of the eigenvectors, Dey et al. [9. 10] proposed a scattering-type parameter $\theta_{\mathrm{FP}}$, and a scattering asymmetry parameter (i.e., helicity) $\tau_{\mathrm{FP}}$ for full polarimetric (FP) SAR data. The method directly utilizes the coherency matrix elements (or the Kennaugh matrix elements) and the Barakat degree of polarization [11]. This method does not utilize the eigendecomposition of the coherency matrix and relates the Huynen parameters [12] to characterize target information. Dey et al. similarly extended the notion of $\theta_{\mathrm{FP}}$ to dual co-polarimetric (i.e., HH-VV) and compact polarimetric (i.e., RH-RV or LH-LV) SAR data and utilized them along with $\tau_{\mathrm{FP}}$, and $H$ for many applications [13, 14].

The degree of polarization has been widely utilized in radar polarimetry and is considered the most crucial parameter that describes a partially polarized electromagnetic wave [15]. This scalar quantity can be obtained by power measurements using the Stokes vector formalism and can be expressed as a combination of the degree of linear and circular polarization. 
The degree of polarization characterizes the polarimetric purity of plane waves. It represents the correlation between the orthogonal components of the electric field. The polarization state of an electromagnetic field is described using the $2 \times 2$ covariance matrix or the Stokes vector [16]. A pure polarization state corresponds to a rank one matrix with the degree of polarization being 1 , whereas, for a pure depolarized state, the degree of polarization is 0 . It is invariant under unitary transformations (such as rotation) and hence invariant of the receiver polarization basis.

The 2D formalism was further investigated for optical fields represented by $3 \times 3$ coherency matrix. The degree of polarization is formulated with a few scalar invariants (i.e., the determinant and traces of different powers of the coherency matrix). Barakat [11,17] proposed two measures for the degree of polarization, out of which one is similar to the formulation independently proposed by Samson [18].

Polarization characteristics of arbitrary electromagnetic waves have been studied in terms of the entropy [19], and generalized spectral-density Stokes parameters [20]. Moreover, it has been well studied in literature and has been explicitly reported that the $3 \times 3$ coherency matrix, in general, cannot be decomposed into fully polarized and fully unpolarized states like the $2 \mathrm{D}$ case. Therefore, a straightforward insight into the general formalism of the degree of polarization for arbitrary dimensions is challenging.

Luis [21] provided an alternative approach in defining the degree of polarization as a distance measure between the correlation matrix of the electric field and the identity matrix representing completely unpolarized waves. Gil et al. [22, 24] proposed a set of indices (indices of purity) characterizing polarimetric purity of light and material media utilizing the eigenvalues of the coherency matrix. These two indices provide complete information about the polarimetric purity of the polarization state of a wave. In the literature, they are referred to as the degree of polarization and degree of directionality. The overall state of polarization is referred to as the degree of purity [23].

However, one must exercise diligence to characterize these quantities because, in radar polarimetry, the $2 \times 2$ covariance matrix corresponds to wave polarimetry. In contrast, the $3 \times 3$ coherency (covariance) matrix corresponds to scattering polarimetry, unlike in optical fields where the $3 \times 3$ matrix represents the covariance structure among the three components of the electric field (i.e., $E_{x}, E_{y}$, and $E_{z}$ ). Therefore, one must distinctly interpret the concept of waves and media while employing them. Nevertheless, the Barakat degree of polarization has been used in the study of full polarimetric SAR data for some applications [9, 10, 25, 28].

The analyses above stem from considering the characteristics of electromagnetic waves propagation. Another path is possible and has been seldom explored in the literature: Information-Theoretic tools such as Entropy and Complexity. Entropy and complexity are valuable descriptors of a complex system, e.g., those stemming from the chaotic behavior of natural phenomena such as meteorological observations. Entropy is a measure of the unpredictability of the system and relates solely to the probability distribution of its states. The system has minimum entropy when it exhibits only one state with probability one and maximum entropy when all possible states are equiprobable. Complexity relates to the correlation structure of the outcomes, i.e., it describes relationships among the system's components. Quoting Feldman and Crutchfield [29]:

[...] the larger and more intricate the "correlations" between the system's constituents, the more structured its underlying distribution. Structure and correlation are not completely independent of randomness, however. It is generally agreed that both maximally random and perfectly ordered systems possess no structure. Nevertheless, at a given level of randomness away from these extremes, there can be an enormously wide range of differently structured processes.

By following similar concepts, we introduce two parameters in radar polarimetry: (i) polarimetric scattering purity, and (ii) polarimetric scattering complexity using the matrix condition number bounds. These two parameters provide complementary information about targets from polarimetric SAR data in addition to the existing classical descriptors in the literature of radar polarimetry, such as, $\bar{\alpha}[2], \alpha_{s}$ [4], $\alpha_{\mathrm{GD}}[8]$, and $\theta_{\mathrm{FP}}[9,10]$ providing scattering-type information, and the entropy $H$ [2] providing scattering randomness information.

In order to express the polarimetric purity and complexity parameters, we first propose an alternate expression for the generalized degree of polarimetric purity in Section II-A Then, we utilize this expression to adopt these two seemingly distinct concepts: polarimetric purity and complexity measures to analyze full polarimetric SAR data. We propose the polarimetric purity measure in Section II-B using the inequalities relating to the bounds of the matrix condition number, which is suitably associated with the pedestal height in radar polarimetry.

We propose a measure of polarimetric complexity in Section II-C which describes the mixture of orthogonal canonical targets. Like the purity measure, we formulated the complexity measure using the inequalities relating to the bounds of the matrix condition number and the degree of polarimetric purity for equiprobable contributions of orthogonally polarized pure components. We deal with the category of complexity that is small for pure (coherent) and random targets, with a maximum for the equal probable mixture of orthogonal targets.

Section II-D illustrates these measures with examples using mixtures of canonical targets and multiple scattering with varying order. Furthermore, in Section III we utilize full polarimetric C- and L-band data to critically analyze these two parameters for (i) spatial variation over diverse land cover classes and (ii) temporal variation over rice crop. Finally, in Section [V] we conclude by emphasizing the significance and relevance of the two proposed parameters to characterize full polarimetric SAR data.

\section{Methodology}

\section{A. An Alternate Expression for the Generalized Degree of Polarimetric Purity}

Polarimetric purity relates to how close a polarization state of a wave is to its completely polarized component. In the case 
of 2D states of polarization, the Poincáre sphere provides a convenient representation utilizing the elements of the Stokes vector. The Hermitian positive semi-definite $2 \times 2$ covariance matrix completely specifies the state of polarization of the planar field. The degree of polarization can be expressed as the ratio of the intensity (i.e., trace of the covariance matrix) of the fully polarized wave to the total intensity of the wave.

The generalized degree of polarimetric purity $P_{n D}$ for the $n \times n$ coherency matrix $\boldsymbol{\Phi}$ is defined as [22],

$$
P_{n D}=\left\{\frac{1}{n-1}\left[\frac{n\left[\operatorname{tr}\left(\boldsymbol{\Phi}^{2}\right)\right]}{(\operatorname{tr} \boldsymbol{\Phi})^{2}}-1\right]\right\}^{1 / 2}
$$

where $\operatorname{tr}(\boldsymbol{\Phi})$ is the trace of $\boldsymbol{\Phi}$. This is a rotation invariant non-dimensional quantity satisfying $0 \leq P_{n D} \leq 1$. The minimum value of the degree of purity $P_{n D}=0$ corresponds to a completely random polarization state. In contrast, the maximum value $P_{n D}=1$ corresponds to a completely pure polarization state.

In radar polarimetry, the polarization state of the received wave is characterized by $n \times n$ covariance (or coherency) matrices $\boldsymbol{\Phi}$, which contains all physical and measurable information of the target [30].

Thus, for full-polarimetric monostatic SAR data, $\boldsymbol{\Phi}$ is a $3 \times 3$ Hermitian positive semi-definite coherency matrix $\mathbf{T}$, or the covariance matrix $\mathbf{C}$. For dual-polarimetric SAR data, $\mathbf{\Phi}$ is a $2 \times 2$ Hermitian positive semi-definite covariance matrix $\mathrm{C}_{2}$.

The mean $(m)$ and the standard deviation $(s)$ of the real positive eigenvalues $\lambda_{1} \geq \lambda_{2} \geq \cdots \geq \lambda_{n} \geq 0$ for a $n \times n$ Hermitian positive semi-definite matrix $\Phi$ are defined using a simple function of the trace of the matrix and the trace of its square given in [31] as,

$$
\begin{aligned}
m & =\frac{1}{n} \sum_{j=1}^{n} \lambda_{j}=\frac{1}{n} \operatorname{tr}(\boldsymbol{\Phi}), \text { and } \\
s^{2} & =\frac{1}{n}\left[\sum_{j=1}^{n} \lambda_{j}^{2}-\frac{1}{n}\left(\sum_{j=1}^{n} \lambda_{j}\right)^{2}\right]=\frac{\operatorname{tr}\left(\boldsymbol{\Phi}^{2}\right)-(\operatorname{tr} \boldsymbol{\Phi})^{2} / n}{n} \\
& =\frac{\operatorname{tr}\left(\boldsymbol{\Phi}^{2}\right)}{n}-m^{2}
\end{aligned}
$$

By suitably using these two quantities, we propose a new expression for the generalized degree of polarimetric purity as,

$$
P_{n D}=\frac{s}{\sqrt{n-1} m}
$$

One can easily observe that for $n=2$ and $n=3$, the expression for the generalized degree of polarimetric purity becomes, respectively:

$$
\begin{aligned}
& P_{2 D}=\left\{\frac{2\left[\operatorname{tr}\left(\boldsymbol{\Phi}^{2}\right)\right]}{(\operatorname{tr} \boldsymbol{\Phi})^{2}}-1\right\}^{1 / 2}=\frac{s}{m}, \text { and } \\
& P_{3 D}=\left\{\frac{1}{2}\left[\frac{3\left[\operatorname{tr}\left(\boldsymbol{\Phi}^{2}\right)\right]}{(\operatorname{tr} \boldsymbol{\Phi})^{2}}-1\right]\right\}^{1 / 2}=\frac{s}{\sqrt{2} m} .
\end{aligned}
$$

Therefore, unlike the conventional expression (1) presented in many works of literature, the proposed expression (5) is physically intuitive as it directly relates the measure of scattering purity to the coefficient of variation (i.e., $s / m$ ) of the eigenvalues of a $n \times n$ matrix.

In the following sections using this alternative expression for the generalized degree of polarimetric purity $P_{n D}$, we introduce two parameters in radar polarimetry: (i) polarimetric scattering purity, and (ii) polarimetric scattering complexity. We utilize certain inequalities on the condition number bounds for Hermitian positive definite matrices defined in terms of $m$ and $s$ to derive these two quantities.

\section{B. Polarimetric Scattering Purity}

For a $n \times n$ Hermitian positive definite matrix $\boldsymbol{\Phi}$, the upper and lower bounds of the matrix condition number $(\kappa(\boldsymbol{\Phi})=$ $\left.\lambda_{\max }(\boldsymbol{\Phi}) / \lambda_{\min }(\boldsymbol{\Phi}) \geq 1\right)$ is given in [31] as,

- Upper bound

$$
\kappa(\boldsymbol{\Phi}) \leq 1+\frac{(2 n)^{1 / 2} s\left[m+s /(n-1)^{1 / 2}\right]^{n-1}}{\operatorname{det}(\boldsymbol{\Phi})}
$$

- Lower bound

$$
\begin{cases}1+\frac{2 s}{m-s /(n-1)^{1 / 2}} \leq \kappa(\boldsymbol{\Phi}), & n \text { is even } \\ 1+\frac{2 s n /\left(n^{2}-1\right)^{1 / 2}}{m-s /(n-1)^{1 / 2}} \leq \kappa(\boldsymbol{\Phi}), & n \text { is odd }\end{cases}
$$

where $\lambda_{\max }$ and $\lambda_{\min }$ are the maximum and minimum eigenvalues of $\boldsymbol{\Phi}$ respectively, and $\operatorname{det}(\boldsymbol{\Phi})$ is the determinant of $\Phi$.

Broadly speaking, the condition number measures the sensitivity of the output argument to the changes in the input value. A well-conditioned problem does not change its output argument with minor changes in the input argument, while it is vice-versa for ill-conditioned problems. For a linear equation, the condition number provides error bounds on the solution after approximation. Chaos is also characterized by the sensitivity of a deterministic system to the input parameters. We may say that a system is chaotic when minute variations in the initial conditions produce very different states. In this regard, the condition number and chaotic behavior describe similar properties.

An equivalent definition of matrix condition number is given as, $\kappa(\boldsymbol{\Phi})=\|\boldsymbol{\Phi}\|\left\|\boldsymbol{\Phi}^{-1}\right\|$, where $\|\boldsymbol{\Phi}\|=\max (\|\boldsymbol{\Phi} \mathbf{x}\| /\|\mathbf{x}\|)$, where $\|\cdot\|$ represents the Euclidean norm, and $\mathbf{x}$ is a vector. If a matrix is singular, then its condition number is infinite. A finite large condition number indicates that the matrix is close to being singular.

In the context of radar polarimetry, it can be noted that the inverse of the condition number (i.e., $\lambda_{\min }(\boldsymbol{\Phi}) / \lambda_{\max }(\boldsymbol{\Phi})$ ) is equivalent to the pedestal height obtained from the copolarimetric signature for full polarimetric SAR data [32,33].

The pedestal height represents the ratio of the minimum co-polarization power to the maximum co-polarization power. Alternatively, it is related to the amount of depolarization of the scattered signal (i.e., the generation of partially polarized (polarized plus unpolarized) scattered wave from a scattering target for a completely polarized transmit wave). 
In general, scattering from targets with high surface roughness, such as rough soil surface or targets with randomly oriented distributed structures, such as vegetation, acts as a depolarizing media for the scattered wave.

Therefore, we propose a general measure of polarimetric scattering purity using the matrix condition number. In order to obtain an expression for this quantity, we define two auxiliary descriptors, $0 \leq P_{U} \leq 1$ and $0 \leq P_{L} \leq 1$ quantifying maximum and minimum polarization measures respectively as,

$$
P_{U}=\frac{\kappa_{U}(\mathbf{\Phi})-1}{\kappa_{U}(\mathbf{\Phi})+1}
$$

and

$$
P_{L}=\frac{\kappa_{L}(\boldsymbol{\Phi})-1}{\kappa_{L}(\boldsymbol{\Phi})+1},
$$

where $\kappa_{U}(\boldsymbol{\Phi})$ and $\kappa_{L}(\boldsymbol{\Phi})$ indicate equality in the upper and lower bounds (for $n$ is either even or odd) for the condition number of $\boldsymbol{\Phi}$ respectively.

Using these two descriptors, we propose the polarimetric scattering purity measure, $0 \leq \mathcal{P}_{a}^{(n)} \leq 1$ as,

$$
\mathcal{P}_{a}^{(n)}=\sqrt{\frac{P_{U}^{2}+P_{L}^{2}}{2}} .
$$

One can easily note that for $n=2, \operatorname{det}(\boldsymbol{\Phi})=m^{2}-s^{2}$, and from Equations (6), and (7), we also find that the upper and lower bounds coincide (i.e., $\left.P_{U}=P_{L}\right)$, with $\kappa(\boldsymbol{\Phi})=(m+$ $s) /(m-s)$.

Hence, one can note that $\mathcal{P}_{a}^{(2)}=P_{2 D}=s / m$ is equal to the conventional degree of polarization [15, 16]. However, for $n>2, \mathcal{P}_{a}^{(n)}$ characterizes the amount of overall polarization structure in the scattered wave. Like the generalized degree of polarimetric purity, this measure is also invariant under unitary transformations (such as rotation).

This work will mainly consider the case for $n=3$, which corresponds to the coherency (covariance) matrix for fullpolarimetric SAR data. Nevertheless, one can obtain the purity measure for any $n \times n$ Hermitian positive definite matrix $\mathbf{\Phi}$.

\section{Polarimetric Scattering Complexity}

The subject of discussion about "complexity" has engrossed the scientific community for the last few decades. There exist several complexity measures in dynamical system behavior and time series analysis.

The concept of complexity has been perceived at different levels of abstraction. It is assumed that the complexity of a system depends on the number of its components (e.g., types and characteristics of scattering targets in radar polarimetry) and associations among them.

Simple physical systems are either deterministic or random. The former admits an uncomplicated functional description, while the latter are characterized by their first-order statistical properties. In polarimetric SAR imaging, canonical targets (no randomness and zero entropy) belong to the first class, while fully developed speckle (no structure and maximum entropy) belongs to the last. The scattering from mixtures of such simple cases is heterogeneous and more complex.
In radar polarimetry, Cloude and Pottier [2] presented a typical description of such association by combining scattering entropy $(H)$ and anisotropy $(A)$ to characterize one, two, or random scattering mechanisms. However, ambiguities remain in describing the amount of mixture among orthogonal scattering targets.

Here, we define the polarimetric scattering complexity as a measure that describes a mixture of orthogonal polarized pure components in the scattered wave from a target. If the scattered wave is either completely coherent or completely random, the target under consideration is straightforward, and hence the measure of polarimetric scattering complexity is null.

Interestingly, one can compare this quantity with the generalized statistical complexity measure proposed in [34, 35]. This quantity is a function that captures order (or disorder) and a distance to an equilibrium distribution [36]. The former is computed by a measure of entropy, while the latter depends on the definition of a stochastic divergence.

In this work, similar to the generalized statistical complexity measure, we also deal with the category of complexity that is small for pure (coherent) and random targets, with a maximum for the equal probable mixture of orthogonal targets.

We express the polarimetric scattering complexity measure, $0^{\circ} \leq \mathcal{C}^{(n)} \leq 45^{\circ}$ by using the two auxiliary descriptors, $P_{U}$, and $P_{L}$ (defined earlier) as,

$$
\mathcal{C}^{(n)}=\tan ^{-1}\left(d^{(n)} / d_{\max }^{(n)}\right)
$$

where

$$
d^{(n)}=\left(P_{U}-P_{L}\right) / 2, \quad 0 \leq d^{(n)} \leq 1 .
$$

For $n=2, P_{U}=P_{L}$, and hence, $\mathcal{C}^{(2)}=0^{\circ}$. However, for $n=3$, it is shown in [24] that $P_{3 D}=1 / 2$ for equiprobable contributions of two orthogonally polarized pure components with equal scattering magnitudes. Therefore, one can easily show that for this case, $d_{\max }^{(3)}=1 / 5$.

Similarly, for $n=4$, it has been also shown in [24 that $P_{4 D}=1 / \sqrt{3}$ for equiprobable contributions of two orthogonally polarized pure components with equal scattering magnitudes, for which $d_{\max }^{(4)}=(\sqrt{3}-1) /(4 \sqrt{3}-2)$.

Additionally, $P_{4 D}=1 / 3$ for equiprobable contributions of three orthogonally polarized pure components with equal scattering magnitudes, for which $d_{\max }^{(4)}=1 /(2+\sqrt{3})$. In the following section, we explain the proposed measures using two examples for $n=3$.

\section{Examples of $\mathcal{P}_{a}^{(3)}$ and $\mathcal{C}^{(3)}$}

In this section, we provide some insights into the variations of $\mathcal{P}_{a}^{(n)}$ and $\mathcal{C}^{(n)}$ using a few typical targets represented by $3 \times$ $3 \mathbf{T}$ matrices in radar polarimetry. We consider two different cases to illustrate the two proposed measures.

1) Mixture of canonical targets: In this section, we analyze the variations $\mathcal{P}_{a}^{(3)}$ and $\mathcal{C}^{(3)}$ for the incoherent mixture of two targets defined by their coherency matrices, $\mathbf{T}_{a}$ and $\mathbf{T}_{b}$ as,

$$
\mathbf{T}_{f}=(1-f) \cdot \mathbf{T}_{a}+f \cdot \mathbf{T}_{b}
$$


where $f \in[0,1]$ defines the mixture proportion. One can observe from Fig. 1, that for the mixture of a trihedral target $\left(\mathbf{T}_{a}\right)$, giving odd-bounce scattering, and a dihedral target $\left(\mathbf{T}_{b}\right)$, giving even-bounce scattering, $\mathcal{P}_{a}^{(3)}=1$ and $\mathcal{C}^{(3)}=0^{\circ}$ for $f=0$ and $f=1$ respectively.

However, it is interesting to note that $\mathcal{P}_{a}^{(3)}=0.82$ and $\mathcal{C}^{(3)}=45^{\circ}$ for $f=0.5$. This observation suggests that the scattering complexity reaches its maximum for an equal mixture of two orthogonal canonical targets [37, 38]. In contrast, the scattering purity slightly decreases from the maximum for this equal mixture proportion. A similar result is also true for the mixture of a trihedral and a helix target.
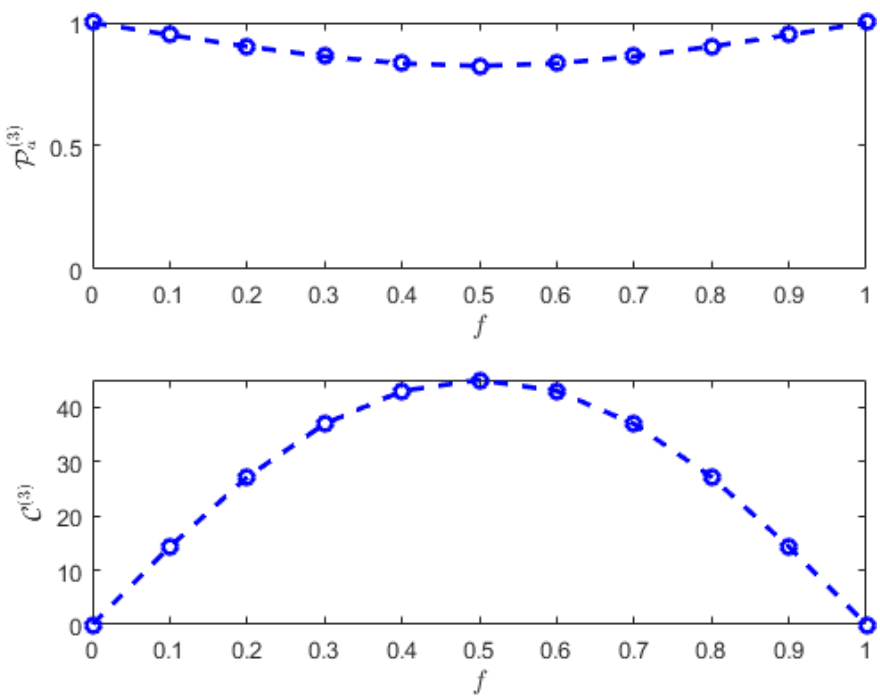

Fig. 1. $\mathcal{P}_{a}^{(3)}$ and $\mathcal{C}^{(3)}$ for a mixture of trihedral and dihedral targets.

The $\mathcal{C}^{(3)}$ vs. $\mathcal{P}_{a}^{(3)}$ plot in Fig. 2 also shows the variation among them with $f$. Overall, one can observe that in the case of a mixture of canonical targets, the variation of $\mathcal{P}_{a}^{(3)}$ and $\mathcal{C}^{(3)}$ with the fraction $f$ reveals their typical response for pure targets and their mixtures with varying proportions. For example, $\mathcal{P}_{a}^{(3)}=1$ and $\mathcal{C}^{(3)}=0^{\circ}$ for $f=0$ and $f=1$ respectively, whereas $\mathcal{C}^{(3)}=45^{\circ}$ for $f=0.5$, i.e., perfectly equal mixture of two orthogonal mechanism [37, 38].

One can observe from Fig. 3. that $\mathcal{P}_{a}^{(3)}=1$ and $\mathcal{P}_{a}^{(3)}=0.33$ for $f=0$ and $f=1$, respectively for the mixture of a trihedral $\left(\mathbf{T}_{a}\right)$ and the random volume $\left(\mathbf{T}_{b}\right)$ [39]. It is interesting to note that for $f=1$ (i.e., complete random volume target), the overall polarization purity, although low, nevertheless persists.

Similarly, $\mathcal{C}^{(3)}=0^{\circ}$ and $\mathcal{C}^{(3)}=13.13^{\circ}$, for $f=0$ and $f=1$, respectively. The maximum value of $\mathcal{C}^{(3)}=27.71^{\circ}$ reaches for $f=0.5$.

The $\mathcal{C}^{(3)}$ vs. $\mathcal{P}_{a}^{(3)}$ plot in Fig. 4 also shows the variation among them with $f$. It is apparent that unlike the mixture of two orthogonal canonical targets, the mixture of trihedral and random volume characterizes the variability in the polarization structure with moderate complexity.

2) Multiple scattering: In this section, we analyze the variations $\mathcal{P}_{a}^{(n)}$ and $\mathcal{C}^{(n)}$ for backscattering as a function of scattering order $(k)$ from a cloud of identical spherical

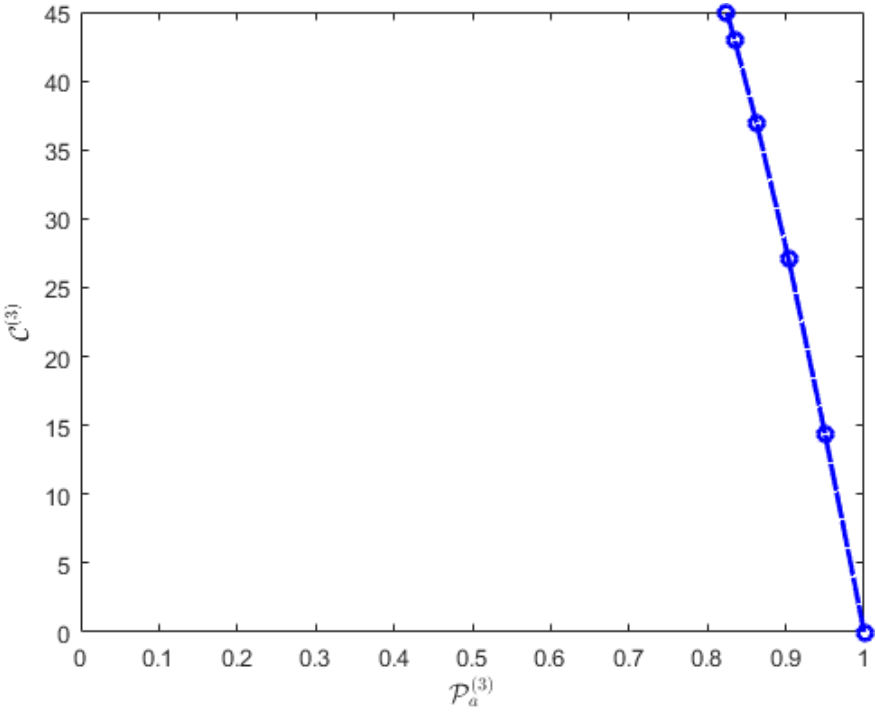

Fig. 2. $\mathcal{C}^{(3)}$ vs. $\mathcal{P}_{a}^{(3)}$ for a mixture of trihedral and dihedral targets.
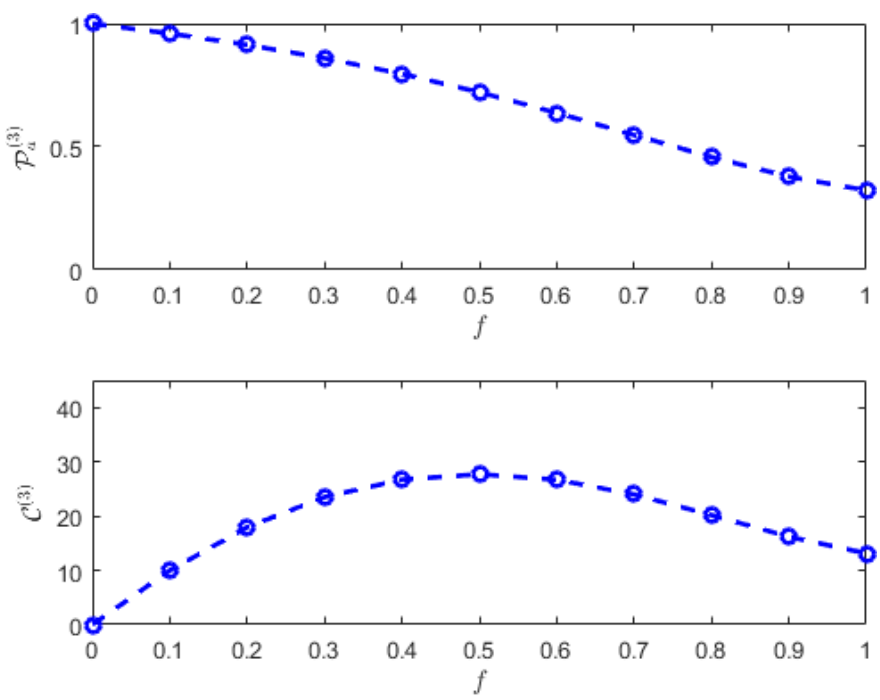

Fig. 3. $\mathcal{P}_{a}^{(3)}$ and $\mathcal{C}^{(3)}$ for a mixture of trihedral and random volume targets.

particles [30,40]. The $3 \times 3$ coherency matrix $\mathbf{T}$ for this medium is given as,

$\mathbf{T}=\left[\begin{array}{ccc}1+\left(\frac{7}{3}+\left(\frac{5}{7}\right)^{k}\right) \chi & 0 & 0 \\ 0 & 1+\left(\frac{1}{3}-\left(\frac{5}{7}\right)^{k}\right) \chi & 0 \\ 0 & 0 & 1+\left(\left(\frac{5}{7}\right)^{k}-\frac{5}{3}\right) \chi\end{array}\right]$,

where $\chi=\frac{3}{2}\left(\frac{7}{10}\right)^{k}$. One can observe that the scattering entropy increases and approaches unity (i.e., an ideal depolarizer) as $k$ increases. Therefore, for large $k$, the target coherency matrix becomes an identity matrix (or its scalar multiple) and represents a completely depolarized signal (or noise).

However, it is interesting to note that significant polarized structures sustain the ensemble averaging process for low order multiple scattering (i.e., for small values of $k$ ). This description of targets provides relevant implications for utilizing polariza- 


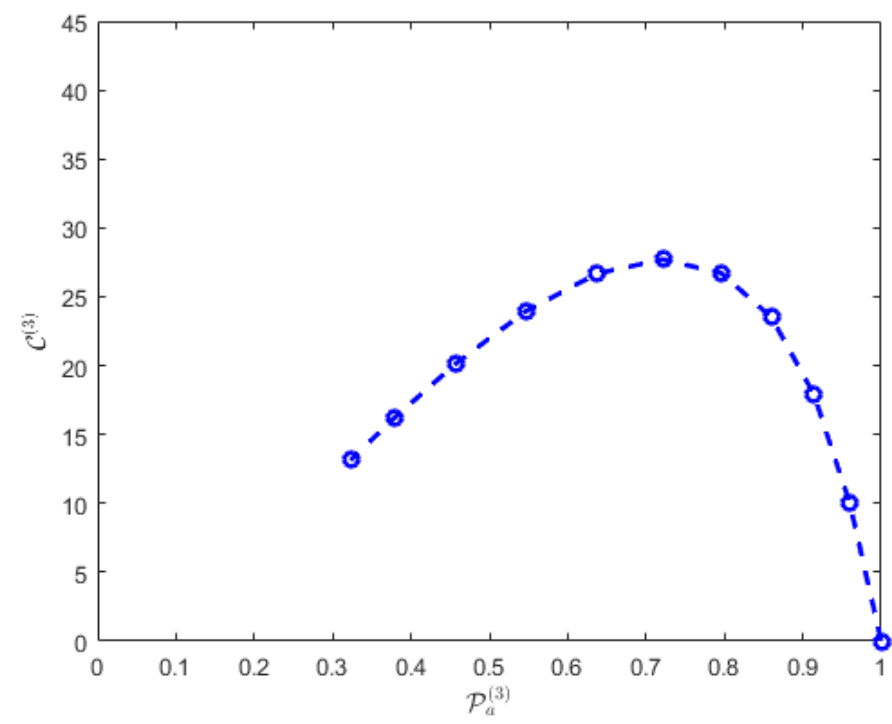

Fig. 4. $\mathcal{C}^{(3)}$ vs. $\mathcal{P}_{a}^{(3)}$ for a mixture of trihedral and random volume targets.

tion in radar remote sensing of distributed targets. Hence, the measure of polarimetric scattering purity and complexity plays a vital role.

In radar polarimetry (i.e., for $n=3$ ), the Barakat degree of polarization is usually used to infer scattering purity [9. 10, 13, 14, 41]. However, one can note that only for $n=2$, the Barakat degree of polarization is equal to the conventional degree of polarization.

For the multiple scattering case given by the above coherency matrix, one can notice in Fig. 5 that for both $k=0$ (i.e., single scattering from a random medium) and $k=1$, the Barakat degree of polarization $P_{3}(3)$ (See Appendix A is unity (i.e., indicating perfect purity). However, the scattering entropy $H$ suggests that for $k=1$, the scattering is partially pure (i.e., $H=0.34$ ).

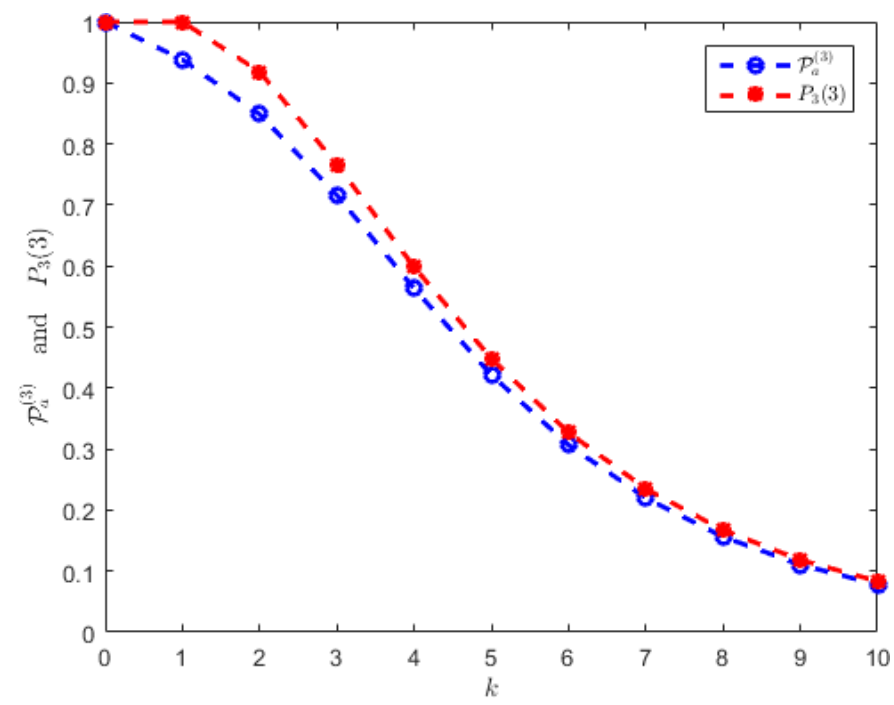

Fig. 5. A comparison of $\mathcal{P}_{a}^{(3)}$ with the Barakat degree of polarization $P_{3}(3)$ for multiple scattering of order $k$.
This inconsistency in the description of scattering is because the Barakat degree of polarization does not quantify the overall degree of polarization, but only a part of it [17] (See Appendix A.

However, one can note that $\mathcal{P}_{a}^{(3)}=1$ for $k=0$, but $\mathcal{P}_{a}^{(3)}=0.94$ for $k=1$. This indicates that the proposed purity parameter $\mathcal{P}_{a}^{(3)}$ precisely describes the overall scattering, unlike the Barakat degree of polarization.
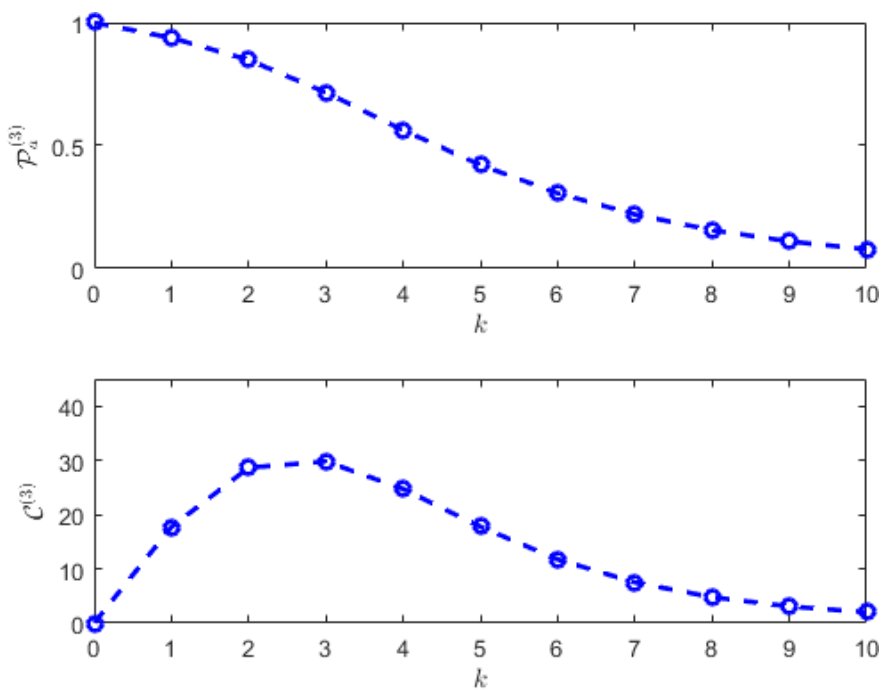

Fig. 6. $\mathcal{P}_{a}^{(3)}$ and $\mathcal{C}^{(3)}$ for multiple scattering of order $k$.

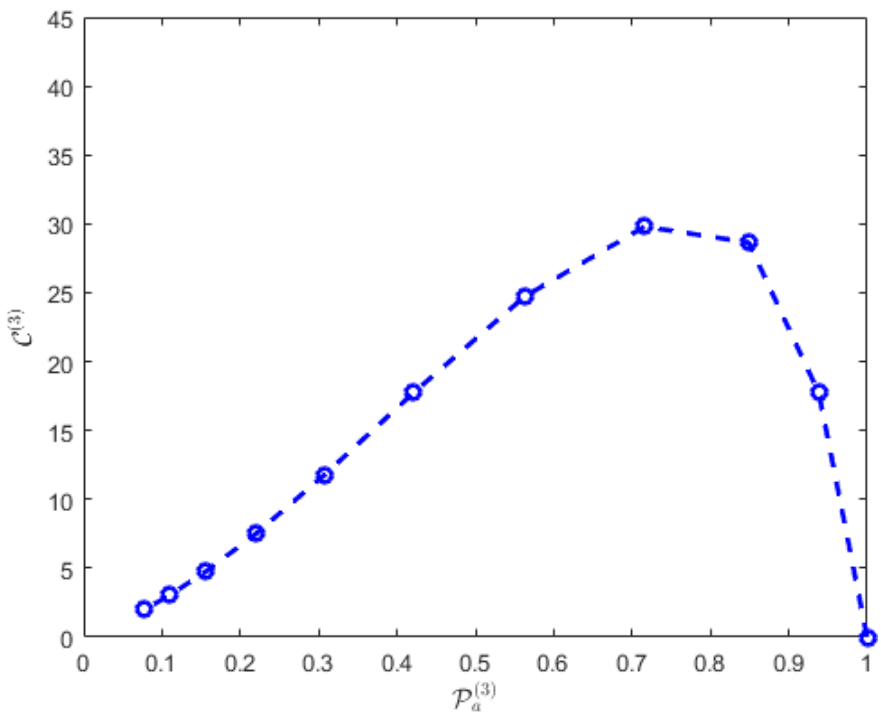

Fig. 7. $\mathcal{C}^{(3)}$ vs. $\mathcal{P}_{a}^{(3)}$ for multiple scattering of order $k$.

Nevertheless, one can observe from Fig. 6 that similar to the Barakat degree of polarization, $\mathcal{P}_{a}^{(3)}$ decays smoothly with increasing $k$. It is interesting to note the variation of $\mathcal{C}^{(3)}$ with $k$. The maximum value of $\mathcal{C}^{(3)}=29.79^{\circ}$ is attained for $k=3$. The right-skewed plot justifies possible mixtures of orthogonally polarized scattering components that exist for the lower order of scattering with its maximum attaining at $k=3$. 
The $\mathcal{C}^{(3)}$ vs. $\mathcal{P}_{a}^{(3)}$ plot in Fig 7 presents a precise depiction of the relationship of their variation.

\section{RESULTS}

In Section $[\mathrm{I}$ we derived the two parameters, i.e., scattering purity, $\mathcal{P}_{a}^{(3)}$, and scattering complexity $\mathcal{C}^{(3)}$ for $n \times n$ Hermitian positive semi-definite matrices. We have investigated their variations for a few typical targets in radar polarimetry.

In this section, we utilize (i) full-polarimetric $\mathrm{C}$ - and L-band SAR data to characterize the spatial variation of $\mathcal{P}_{a}^{(3)}$ and $\mathcal{C}^{(3)}$ over various land cover classes and (ii) full-polarimetric Cband SAR data to characterize the temporal variation of $\mathcal{P}_{a}^{(3)}$ and $\mathcal{C}^{(3)}$ over rice crop.

\section{A. Spatial Variation Analysis}

This section analyzes the spatial variation of $\mathcal{P}_{a}^{(3)}$ and $\mathcal{C}^{(3)}$ to characterize scattering behavior from diverse natural and human-made targets using C- and L-band SAR images.

We used two full polarimetric SAR images over SanFrancisco (SF), the USA, and Mumbai, India. The SF scene is acquired by C-band Radarsat- 2 with near and far range incidence angles of $28.02^{\circ}$ and $29.81^{\circ}$, respectively. The Radarsat-2 Single Look Complex (SLC) image is multi-looked by a factor of 2 in the range direction, and 4 in the azimuth direction to generate a $20 \mathrm{~m} \times 20 \mathrm{~m}$ ground pixel.

The spatial variations of $\mathcal{P}_{a}^{(3)}$ and $\mathcal{C}^{(3)}$ are shown in Fig. 8 . One can observe that both parameters are susceptible to the morphological characteristics of the targets. To quantitatively analyze their variations over different targets, we have considered Urban ('U'), Oriented Urban ('OU'), Vegetation ('V'), and Ocean ('O') targets.

It is important to note that we specify a target as urban when the buildings do not have any orientation to the radar line of sight. In contrast, an oriented urban target represents the buildings that have an orientation to the radar line of sight [3].

We show the box plots over the land cover types in Fig. 9 One can observe that over ' $O$ ' the median value of $\mathcal{P}_{a}^{(3)}$ is very high, i.e., $\approx 0.98$, which suggests that the scattering from the ocean surface is relatively pure. This type of scattering from the ocean surface might be due to the calm water surface and more negligible wind effect during acquisition. As a result, the ocean surface scattering resembles a trihedral scatterer characterized by high odd-bounce scattering. A similar phenomenon is also reported in [9].

In contrast, the median value of $\mathcal{C}^{(3)}$ is low, i.e., $\approx 4^{\circ}$. This low value of $\mathcal{C}^{(3)}$ typically indicates that over the ocean surface, only a single dominant scattering mechanism exists along with a negligible volume/diffused scattering. We also analyzed the values of scattering entropy $(H)$ and anisotropy $(A)$ over ' $\mathrm{O}$ ' to confirm this conclusion. Interestingly, we notice that the median value of $(1-H)(1-A)=0.52$ is higher than any other combination of $H$ and $A$ [3] as seen in Table I The values of $H A=0.04, H(1-A)=0.06$ and $(1-H) A=0.38$.

A high median value of $(1-H)(1-A)$ indicates the existence of a single dominant scattering mechanism from the target. By definition, if only one scattering mechanism exists within the resolution cell, $\mathcal{C}^{(3)}=0^{\circ}$. However, in this case, negligible volume/diffused scattering due to small roughness in the ocean surface has marginally increased the value of $\mathcal{C}^{(3)}$. Besides, one should also note that the standard deviation is also very low over this surface, which signifies the homogeneous characteristics of the target.

Over 'U', we observe marginally lower $\mathcal{P}_{a}^{(3)}$ values compared to the ocean surface. This variation indicates that scattering purity over the urban area slightly decreased, which might be due to the geometry of the urban area and azimuth orientations of buildings.

The median value of $\mathcal{P}_{a}^{(3)} \approx 0.8$ over ' $\mathrm{U}$ '. Besides, the standard deviation has also increased due to diverse geometry over the urban region. In contrast, we observe a sudden increase of $\mathcal{C}^{(3)}$ values as compared to the ocean surface. The median value of $\mathcal{C}^{(3)}=40.09^{\circ}$ over ' $U$ '. These high values of $\mathcal{C}^{(3)}$ are likely due to the existence of two orthogonal scattering mechanisms within the resolution cell.

The literature well studied that orthogonal urban areas act as dihedral scatterers, which produces a high amount of evenbounce scattering mechanisms. However, urban areas also produce a substantial amount of odd-bounce mechanisms due to several different geometrical structures. A similar conclusion is also evident from the work of Yamaguchi et al. [42] and Dey et al. [10]. Also, high median values of $H A=0.64$ suggest two main proportions of scattering mechanisms with nearly equal probability as seen in Table I]

We observe a further drop in the median values of $\mathcal{P}_{a}^{(3)}$ over 'OU'. The low values of $\mathcal{P}_{a}^{(3)}$ is due to the high cross-pol component, which is evident over the oriented urban areas [43]. Consequently, the diffused scattering component increases, which in turn decreases the scattering purity measure. The median value of $\mathcal{P}_{a}^{(3)}$ is around 0.60 . In contrast, the values of $\mathcal{C}^{(3)}$ are less as compared to ' $U$ '.

The median value of $\mathcal{C}^{(3)} \approx 29^{\circ}$. Therefore, the drop in the $\mathcal{C}^{(3)}$ value is around $10 \%$ compared to ' $\mathrm{U}$ ', which is due to the high amount of volume/diffused scattering mechanism [10]. Besides, we observe median values of $H(1-A)=0.48$ and $H A=0.41$ in Table I These values most likely indicate that both even-bounce and odd-bounce scattering mechanisms exist within the resolution cell. However, $H(1-A)$ is slightly higher than $H A$, suggesting that the volume/diffused component proportion is almost equal to or higher than even or oddbounce components.

Over vegetation surface, ' $\mathrm{V}$ ' the median value of $\mathcal{P}_{a}^{(3)}$ is 0.49 , which is due to the random scattering from the vegetation surface. In particular, randomly oriented branches, twigs, and leaves are typically considered ensembled of oriented dipoles in a resolution cell. As a result, the scattering entropy is high from these targets, suggesting low values of $\mathcal{P}_{a}^{(3)}$.

Volume (or diffused) scattering component is primarily the dominant mechanism from vegetation targets. However, one commonly observes odd-bounce scattering depending on the leaf and branching structures. The median value of $H(1-$ $A)=0.65$ suggests dominant random scattering, while the median value of $H A=0.17$ suggest a secondary dominant single bounce scattering mechanism. The $\mathcal{C}^{(3)}$ parameter also 


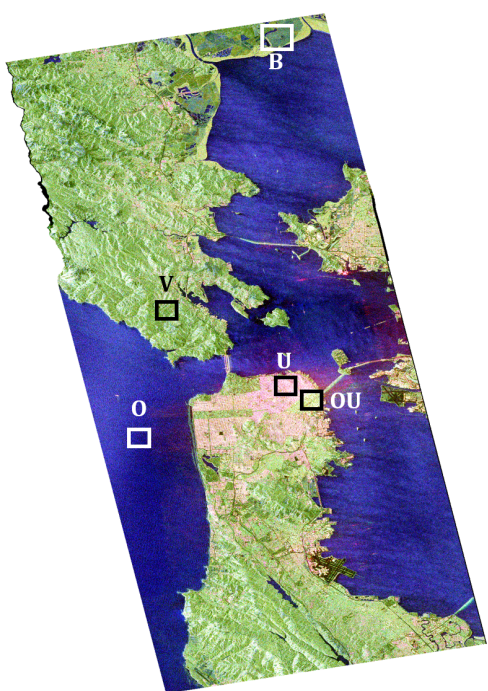

(a) Pauli RGB

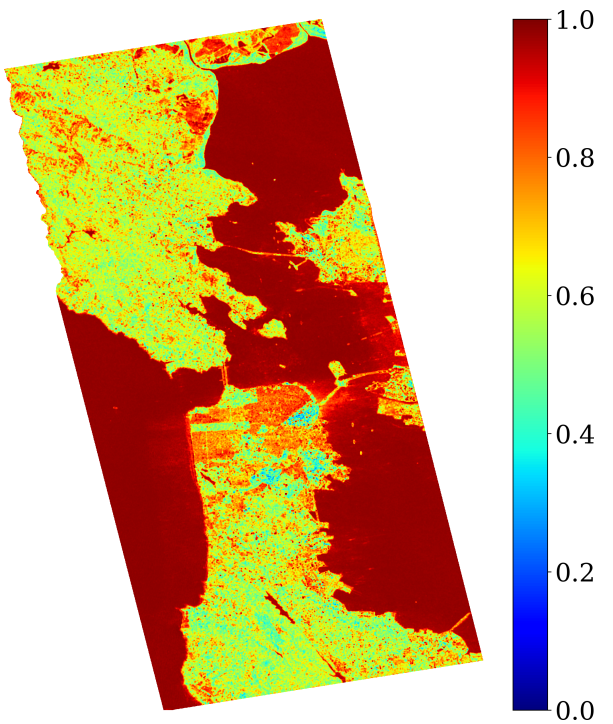

(b) $\mathcal{P}_{a}^{(3)}$

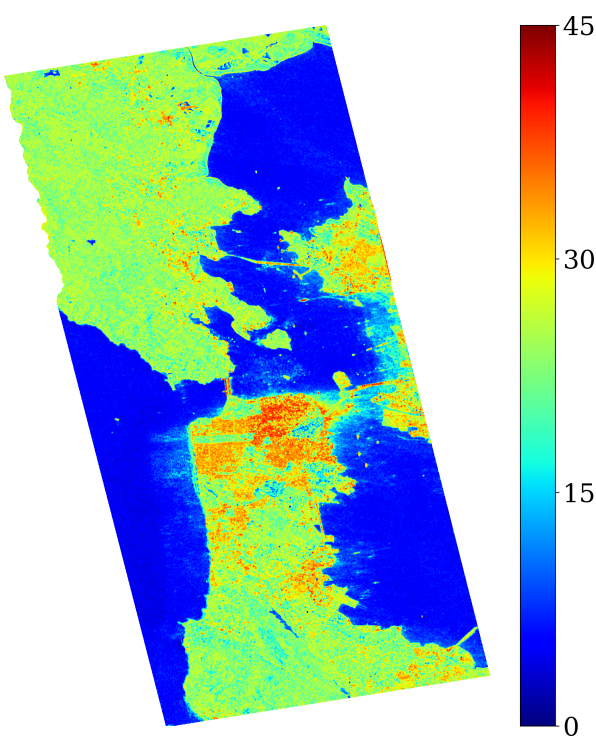

(c) $\mathcal{C}^{(3)}$

Fig. 8. Images of (a) Pauli RGB, (b) $\mathcal{P}_{a}^{(3)}$ and $\mathcal{C}^{(3)}$ for RS-2 FP SAR data over San Francisco. Here, 'O' stands for Ocean, 'U' stands for urban, 'OU' stands for oriented urban, ' $\mathrm{V}$ ' stands for vegetation and ' $\mathrm{B}$ ' stands for barren land with sparse vegetation.

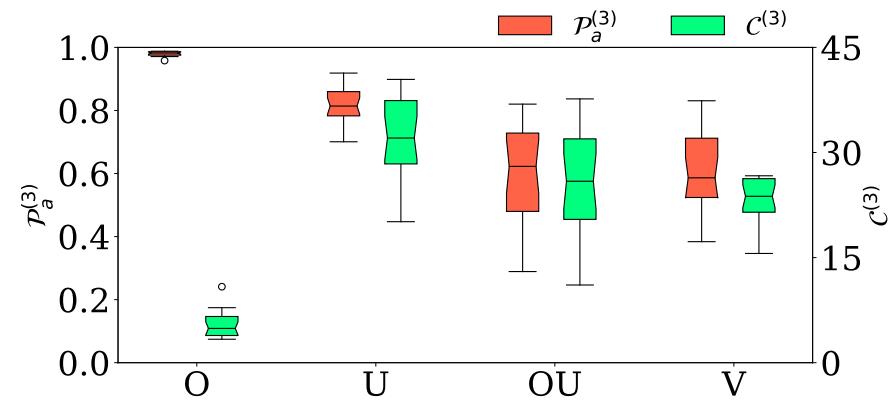

Fig. 9. Variation of $\mathcal{P}_{a}^{(3)}$ and $\mathcal{C}^{(3)}$ over different land cover types over San Francisco Bay area using Radarsat-2 data.

reveals similar properties. We observe lowest values of $\mathcal{C}^{(3)} \approx$ $25^{\circ}$ over vegetation surface.

Over barren fields (B) with sparse vegetation, an interesting trend of $\mathcal{P}_{a}^{(3)}$ and $\mathcal{C}^{(3)}$ is evident. The values of $\mathcal{P}_{a}^{(3)}$ ranges from 0.8 to 0.97 . These values of $\mathcal{P}_{a}^{(3)}$ indicate the scattered wave to be highly pure/polarized. However, the values of $\mathcal{C}^{(3)}$ are not as low as observed over the ocean surface. We observe that the range of $\mathcal{C}^{(3)}$ over this area is $20^{\circ}$ to $24^{\circ}$. These notable values of $\mathcal{C}^{(3)}$ are due to the existence of some volume scattering component along with a strong odd-bounce scattering component over this area.

The Mumbai scene is an L-band ALOS-2 image with a center incidence angle of $33^{\circ}$. The image is multi-looked by a factor of 3 in range direction, and 5 in the azimuth direction to generate $15 \mathrm{~m}$ pixels. The box plots over Ocean ('O'), Urban ('U') and Vegetation ('V') are shown in Fig. 11

We observe interesting differences in scattering characteristics compared with the RS-2 data over various targets, primarily attributed to the difference in the wavelengths. Over
' $\mathrm{O}$ ', the scattering purity is high. However, it is $2 \%$ lower than the value of $\mathcal{P}_{a}^{(3)}$ for RS-2. Therefore, the decorrelation between the $\mathrm{HH}$ and $\mathrm{VV}$ channels over the ocean is higher for the L-band data than C-band data. Consequently, we also observe an increased value of $\mathcal{C}^{(3)}$.

However, similar to RS-2 data, the scattering mechanism over the ocean surface is dominantly odd-bounce. Hence, the scattering complexity is nevertheless lower than any other scattering targets. Moreover, the ocean surface is more homogeneous to L-band data than C-band, due to which the standard deviation is also lower.

Over urban area ' $U$ ', we observe a slight difference in the values of $\mathcal{P}_{a}^{(3)}$ than the ocean surface. The median value of $\mathcal{P}_{a}^{(3)}$ is approximately 0.91 . Furthermore, a big difference between the proportion of even and odd-bounce components is evident. It can be seen from Table II that the values of $H A$, $(1-H) A, H(1-A)$ and $(1-H)(1-A)$ over urban area are $0.62,0.21,0.12$ and 0.04 , respectively. However, in contrast to ' $U$ ' of RS-2 data the median value of $\mathcal{C}^{(3)}=20.20^{\circ}$ is much lower, which states that the orthogonal mixture of scattering mechanisms is lower than RS-2 urban area.

Similar to RS-2, we observe a very low value of $\mathcal{P}_{a}^{(3)}$ over vegetation surface, ' $\mathrm{V}$ ' due to the randomly oriented dipole structures of branches and twigs. Consequently, the value of $\mathcal{C}^{(3)}$ is the lowest among all targets. However, the standard deviation of $\mathcal{P}_{a}^{(3)}$ and $\mathcal{C}^{(3)}$ are quite low over Vegetation, indicating that the vegetation area appears homogeneous to the L-band data during the acquisition. In this case, the value of $H(1-A)=0.80$ is highest among all other $H$ and $A$ combinations. The values of $H A,(1-H) A$, and $(1-H)(1-A)$ over vegetation area are $0.04,0.02$, and 0.14 , respectively as seen in Table $\Pi$ The median values for different combinations of $H$ and $A$ over different land cover

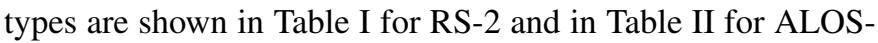




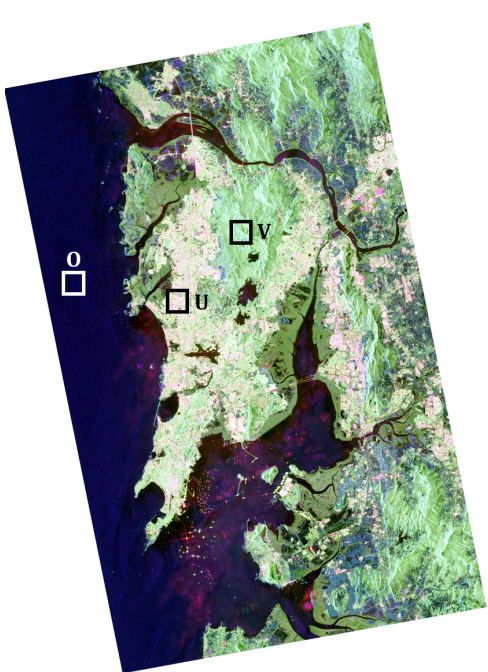

(a) Pauli RGB

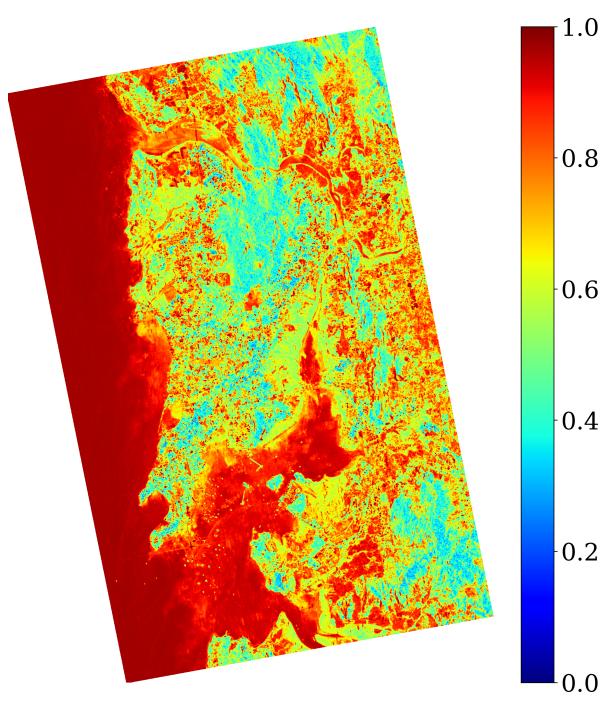

(b) $\mathcal{P}_{a}^{(3)}$

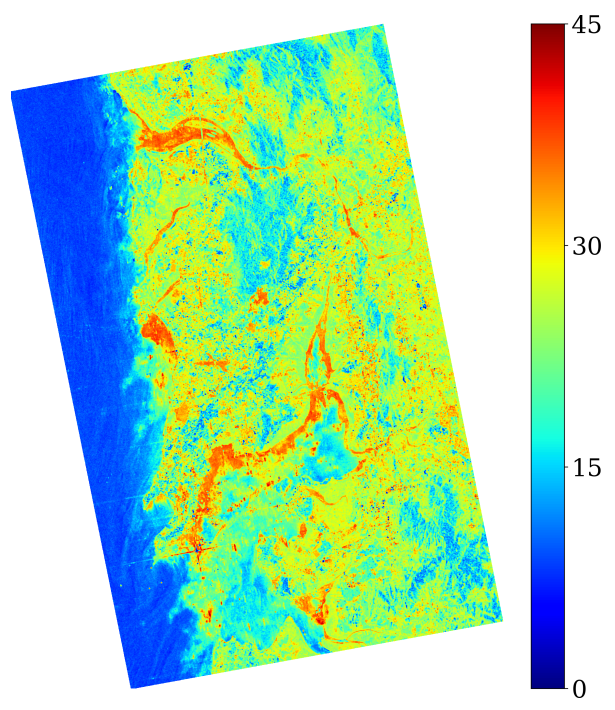

(c) $\mathcal{C}^{(3)}$

Fig. 10. Images of (a) Pauli RGB, (b) $\mathcal{P}_{a}^{(3)}$ and (c) $\mathcal{C}^{(3)}$ for ALOS-2 FP SAR data over Mumbai, India. Here, 'O' stands for Ocean, 'U' stands for urban, and ' $\mathrm{V}$ ' stands for vegetation.

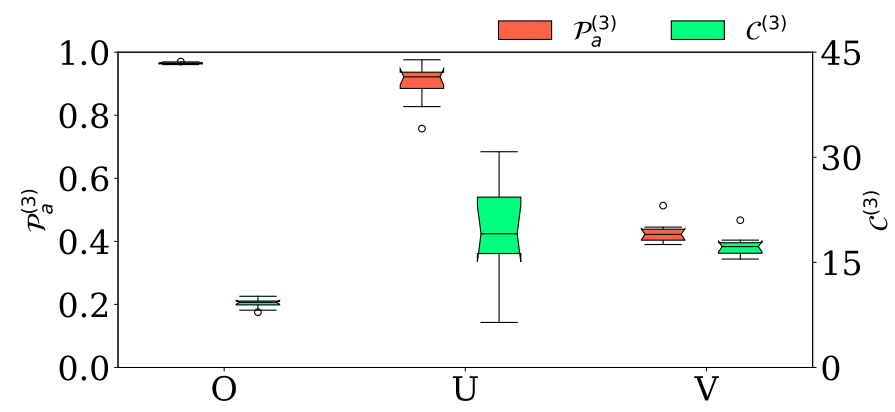

Fig. 11. Variation of $\mathcal{P}_{a}^{(3)}$ and $\mathcal{C}^{(3)}$ over different land cover types over Mumbai, India using the ALOS-2 FP SAR data.

2 data.

TABLE I

MEDIAN VALUES FOR DIFFERENT COMBINATIONS OF ENTROPY $(H)$, ANISOTROPY $(A)$, PURITY $\left(\mathcal{P}_{a}^{(3)}\right)$, AND COMPLEXITY $\left(\mathcal{C}^{(3)}\right)$ OVER DIFFERENT LAND COVER TYPES USING RS-2 DATA

\begin{tabular}{ccccc}
\hline & Ocean & Urban & Oriented Urban & Vegetation \\
\hline$H A$ & 0.04 & $\mathbf{0 . 6 4}$ & $\mathbf{0 . 4 1}$ & 0.17 \\
$H(1-A)$ & 0.06 & 0.10 & $\mathbf{0 . 4 8}$ & $\mathbf{0 . 6 5}$ \\
$(1-H) A$ & 0.38 & 0.23 & 0.05 & 0.04 \\
$(1-H)(1-A)$ & $\mathbf{0 . 5 2}$ & 0.03 & 0.06 & 0.14 \\
\hline $\mathcal{P}_{a}^{(3)}$ & 0.98 & 0.80 & 0.60 & 0.49 \\
$\mathcal{C}^{(3)}\left(^{\circ}\right)$ & 3.73 & 40.09 & 28.60 & 25.33 \\
\hline
\end{tabular}

\section{B. Temporal Variation Analysis}

In this section, we analyze the temporal behavior of $\mathcal{P}_{a}^{(3)}$ and $\mathcal{C}^{(3)}$ over rice crop. Fine Quad Wide (FQW) mode fullpolarimetric Radarsat-2 images were acquired from July to
TABLE II

MEDIAN VALUES FOR DIFFERENT COMBINATIONS OF ENTROPY $(H)$, ANISOTROPY $(A)$, PURITY $\left(\mathcal{P}_{a}^{(3)}\right)$, AND COMPLEXITY $\left(\mathcal{C}^{(3)}\right)$ OVER DIFFERENT LAND COVER TYPES USING ALOS-2 DATA

\begin{tabular}{cccc}
\hline & Ocean & Urban & Vegetation \\
\hline$H A$ & 0.13 & $\mathbf{0 . 6 2}$ & 0.04 \\
$H(1-A)$ & 0.10 & 0.12 & $\mathbf{0 . 8 0}$ \\
$(1-H) A$ & 0.32 & 0.21 & 0.02 \\
$(1-H)(1-A)$ & $\mathbf{0 . 4 5}$ & 0.04 & 0.14 \\
\hline $\mathcal{P}_{a}^{(3)}$ & 0.96 & 0.91 & 0.39 \\
$\mathcal{C}^{(3)}\left(^{\circ}\right)$ & 8.13 & 20.20 & 16.98 \\
\hline
\end{tabular}

November 2018 over the test site, as shown in Table III These images were multi-looked by a factor of $2 \times 3$ pixels in the range and azimuth directions, respectively, to generate $\approx 15 \mathrm{~m} \times 15 \mathrm{~m}$ square pixel images. Following this, we applied a $3 \times 3$ boxcar filter [3] to further reduce speckle. Additionally, the images are then co-registered with a Root Mean Square Error (RMSE) $\leq 0.25 \mathrm{~m}$.

In this study, 40 in-situ field measurements were analyzed. Soil moisture was measured in each field for two sampling locations. These locations were arranged in two parallel transects along the row direction, with a separation between each transect of $\approx 40 \mathrm{~m}$.

The growth stages of rice usually consist of three major phases: vegetative, reproductive, and maturity. The rice cultivation period depends mainly on rainfall events in the test site. The vegetative phase begins with the germination of the seeds and ends with fully developed plants. In this period, the number of tillers increases and stems elongate. The germination phase consists of seed and radicle development.

The onset of the first tiller defines the initiation of the tillering stage. During booting, the upper part of the stem 
TABLE III

RADARSAT-2 DATA ACQUIRED OVER THE JECAM TEST SITE IN VIJAYAWADA, INDIA

\begin{tabular}{|c|c|c|c|c|}
\hline Acquisition date & Beam mode & Incidence angle range $\left(^{\circ}\right)$ & Orbit & azimuth $(\mathrm{m}) \times$ range $(\mathrm{m})$ \\
\hline 05 Jul 2018 & & $33.73-36.65$ & & \\
\hline 29 Jul 2018 & & $33.73-36.65$ & & \\
\hline 22 Aug 2018 & $5015 \mathrm{w}$ & $33.73-36.65$ & A condino & $72 \times 511$ \\
\hline 09 Sep 2018 & FQ15W & $33.73-36.65$ & Ascenaing & $4.13 \times 5.11$ \\
\hline 02 Nov 2018 & & $33.73-36.64$ & & \\
\hline 26 Nov 2018 & & $33.73-36.64$ & & \\
\hline
\end{tabular}

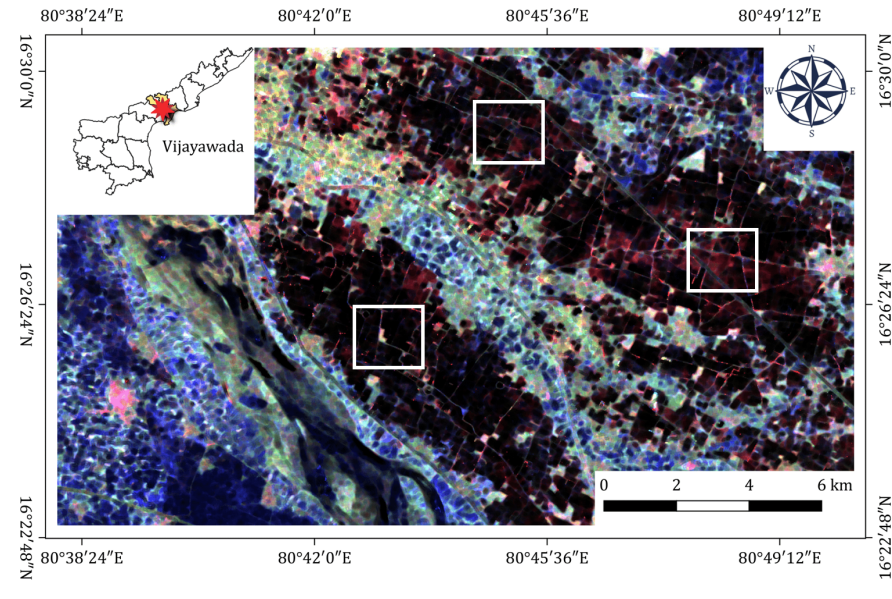

Fig. 12. Pauli RGB image of the JECAM test site over Vijayawada, India acquired on 24 July, 2019. The white boxes indicate the rice cultivation areas.

becomes thick, and flag leaf development begins. The fruit development stage starts when the grain appears and later becomes milky following the complete development of anthers. In the subsequent stage, dough followed by the ripening condition of rice leads to the final harvest.

The temporal variations of $\mathcal{P}_{a}^{(3)}$ and $\mathcal{C}^{(3)}$ over the rice fields are shown in Fig. 13 and Fig. 14, respectively. One can see that both $\mathcal{P}_{a}^{(3)}$ and $\mathcal{C}^{(3)}$ follow a specific pattern over the field depending on the morphological condition of rice at their diverse phenological stages.

It is well understood that the scattering mechanisms from the rice fields depend on the structure of the plant, its branches, the appearance of the flower, and the dielectric property of the stem [13, 14]. Hence, to discuss the changes quantitatively, we presented a box plot of $\mathcal{P}_{a}^{(3)}$ and $\mathcal{C}^{(3)}$ over the rice fields in Fig. 15

It can be observed from Fig. 15 that during 05-Jul the median value of $\mathcal{P}_{a}^{(3)} \approx 0.84$, while median value of $\mathcal{C}^{(3)} \approx 24^{\circ}$. The high value of $\mathcal{P}_{a}^{(3)}$ indicates that the scattered wave from the rice fields is very pure, while the low value of $\mathcal{C}^{(3)}$ suggests the presence of a unique scattering component from the target.

During this period, rice was not cultivated in the fields. However, field preparation started for the initiation of the cultivation process. Therefore, the field condition varied depending on the plowed and non-plowed situation. As a result, notable standard deviation for both $\mathcal{P}_{a}^{(3)}$ and $\mathcal{C}^{(3)}$ are evident in Fig. 15 .

Notably, the EM wave is affected by the soil surface during this time. Hence, a high amount of dominant odd-bounce scattering is evident from the fields [13]. Nevertheless, a significant amount of volume scattering is also apparent due to the increased surface roughness of some fields.

The complexity measurement provides information about the mixture of orthogonal scattering mechanisms. Therefore, in this regard, an odd-bounce scattering is a unique mechanism, and hence, according to the definition, $\mathcal{C}^{(3)}=0^{\circ}$. However, the existence of the volume scattering from the rough fields has slightly increased the value of $\mathcal{C}^{(3)}=24^{\circ}$.

Accordingly, it is essential to note that the odd-bounce scattering and the volume scattering, which are not orthogonal to each other, has the value of $\mathcal{C}^{(3)} \neq 45^{\circ}$, unlike the equiprobable mixture of a trihedral and a dihedral.

On 29-Jul, rice fields reached the early tillering stage. About 1 to 3 tillers appear in the rice canopy during this period, depending on sowing time. At this point, we observe a sudden decline in $\mathcal{P}_{a}^{(3)}$ with its median value of 0.66 . This decrease in $\mathcal{P}_{a}^{(3)}$ could be due to the uneven growth characteristics of rice in all fields. As per in-situ information, most of these fields were non-cultivated due to irrigation limitations or a late sown variety of rice.

Therefore, the high soil surface roughness of specific noncultivated fields might have lowered the values of $\mathcal{P}_{a}^{(3)}$. Moreover, during this period, crop canopy height reached $22.30 \mathrm{~cm}$. As a result, the interaction of the C-band EM wave with the crop canopy has marginally increased the volume/diffused scattering components. This diffused scattering has also impacted the values of $\mathcal{P}_{a}^{(3)}$.

Interestingly, one can observe that although $\mathcal{P}_{a}^{(3)}$ decreased as compared to 05-Jul, $\mathcal{C}^{3}$ increased from the previous date with a median value of $32^{\circ}$. This phenomenon indicates the marginal existence of two orthogonal scattering mechanisms, i.e., odd-bounce and even-bounce, in the resolution cell.

Rice, being a wetland crop, the underlying soil surface was held saturated with water during this period. Moreover, the stem-water interaction of EM waves increases as the rice crop attains a significant height. As a result, the even-bounce scattering mechanism became apparent.

Also, the soil surface produced a substantial proportion of the odd-bounce scattering mechanism. In addition to this, the canopy height and the non-cultivated fields generated volume/diffused scattering, which made the overall mixture 


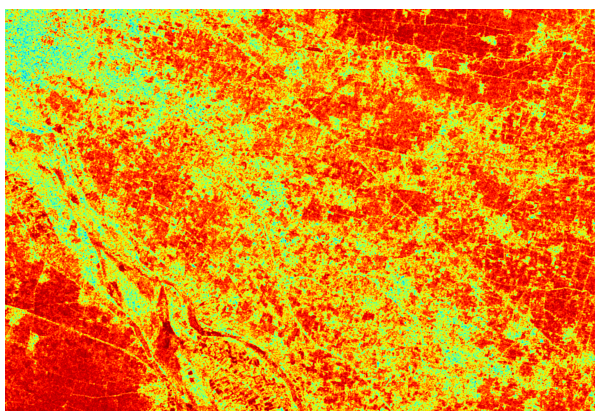

(a) 05-Jul

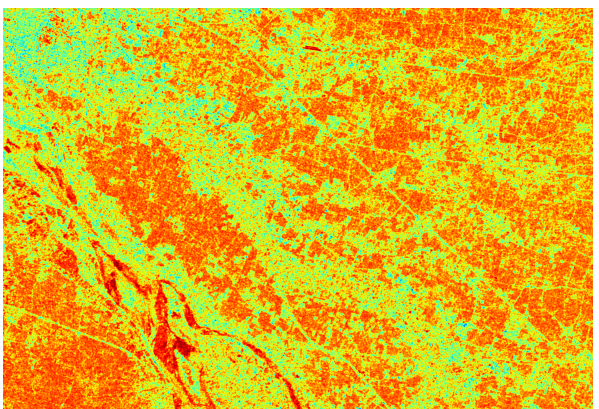

(d) 09-Sep

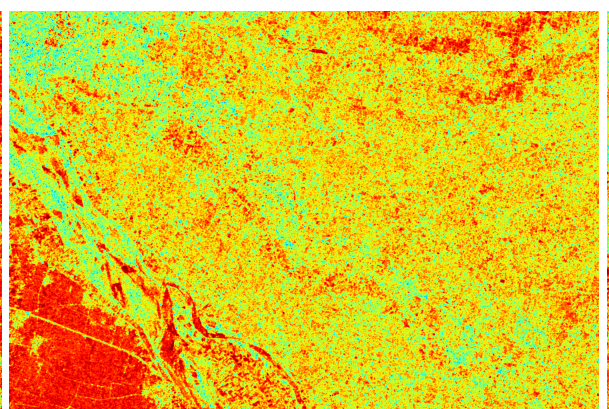

(b) 29-Jul

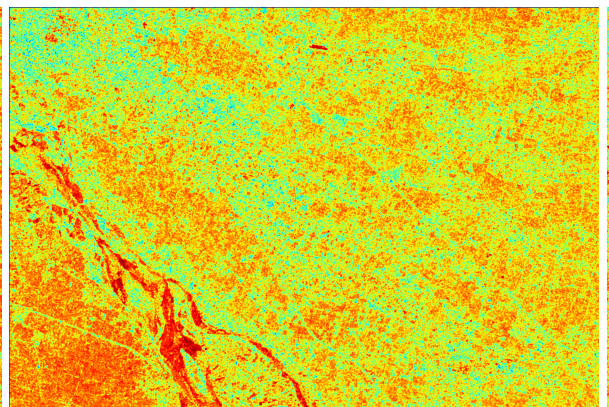

(e) 02-Nov

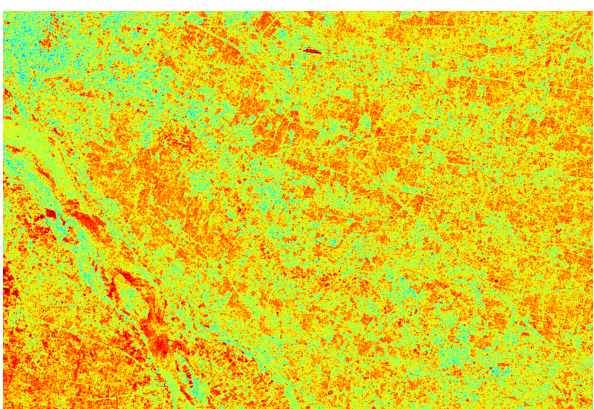

(c) 22-Aug

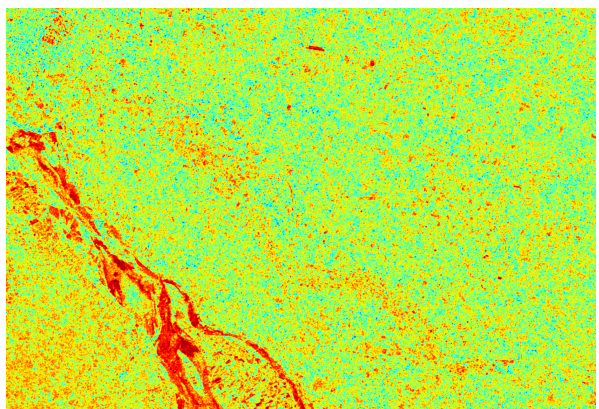

(f) $26-\mathrm{Nov}$

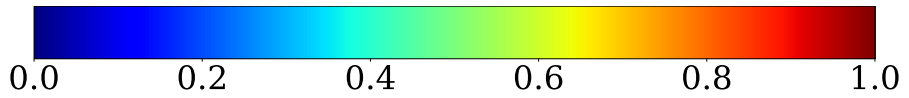

Fig. 13. Time series spatial variation of $\mathcal{P}_{a}^{(3)}$ over different rice phenological stages in Vijayawada, India.

of scattering mechanisms marginally non-unique. Hence, one can observe the increase of $\mathcal{C}^{3}$ by $8^{\circ}$ compared to 05-Jul.

During 22-Aug, most rice fields entered the advanced tillering stage. Moreover, the non-cultivated fields during 29Jul also completed their early tillering stage. Furthermore, this period is the transition between the tillering and stem elongation stages.

At this stage, the inter-node of the rice plant elongates, which in turn increases the height of the stem and subsequently becomes firm. We observe an increased $\mathcal{P}_{a}^{(3)}$ value during this period with its median value of 0.73 . However, the median value of $\mathcal{C}^{(3)}$ remained almost similar, i.e., approximately $33^{\circ}$.

The increase of $\mathcal{P}_{a}^{(3)}$ is due to the increased stem water interaction of the EM wave, which increased the proportion of the even-bounce scattering component. However, the increase in the even-bounce mechanism also resulted in a drastic decrease in the proportion of the odd-bounce scattering mechanism. Hence, even though the value of $\mathcal{P}_{a}^{(3)}$ has increased, we do not observe a significant change in $\mathcal{C}^{(3)}$ values due to the uniqueness of a scattering component in the mixture (i.e., an even-bounce scattering).

On 09-Oct we observe further increase in the $\mathcal{P}_{a}^{(3)}$ values $\approx 0.76$. Most rice fields were in the inflorescence emergence stage with high even-bounce or even multiple bounce scattering during this period. We also observe a significant decrease in the odd-bounce scattering mechanism. The moderate oddbounce scattering might be due to the rice foliage. Therefore, a high imbalance in the proportion of odd-bounce and even- bounce scattering mechanisms has further lowered the $\mathcal{C}^{(3)}$ values to $\approx 29^{\circ}$.

Around $02 \mathrm{Nov}$, the rice fields reached the early dough stage, during which the milky white substance began to accumulate in the rice panicle. Simultaneously, the crop water content during this period remains very high, while leaf and stem produce an overall complex canopy structure, which leads to high randomness in the SAR backscatter. Due to this fact, we observe lower $\mathcal{P}_{a}^{(3)}$ values.

The dense canopy structure increased the volume/diffused scattering mechanism. Also, we observe a marginal increase in the odd-bounce scattering component, while the even-bounce scattering component is reduced drastically. At this time, we observe $2^{\circ}$ decrease in the $\mathcal{C}^{(3)}$ values as compared to 09-Sep.

On $26 \mathrm{Nov}$, the rice fields reached the maturity stage, and the grains became firm and heavy. At this point, the crop becomes dry, whereas the moisture content in grains remains $\approx 20 \%$. Due to the weight of the grains, the lodging of rice is usually visible in the fields, due to which the morphological attribute becomes further complex than the dough stage.

Hence, an additional increase in the scattering entropy during this period is apparent. Moreover, the amount of crosspol components has increased during this period, and the coherence between the co-polarized channels decreased significantly. A similar type of increase in cross-pol components from transplantation to maturity stages was reported by [44]. As a result, both median values of $\mathcal{P}_{a}^{(3)}$ and $\mathcal{C}^{(3)}$ dropped to 0.57 and $27^{\circ}$, respectively. The images of time-series variation 


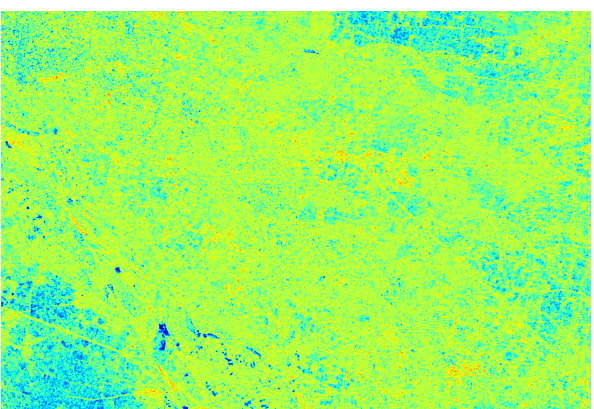

(a) 05-Jul

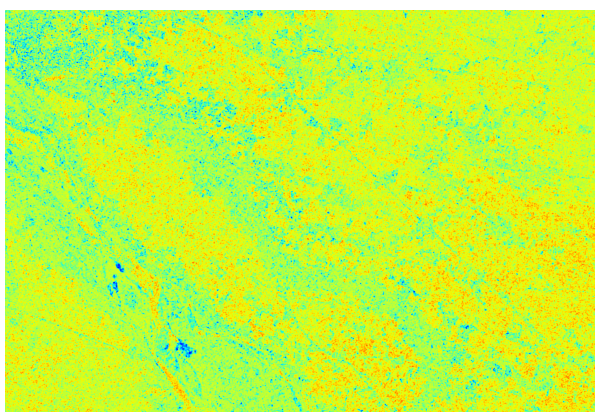

(d) 09-Sep

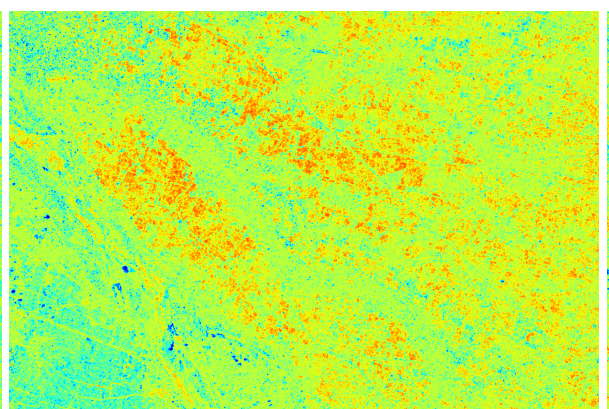

(b) 29-Jul

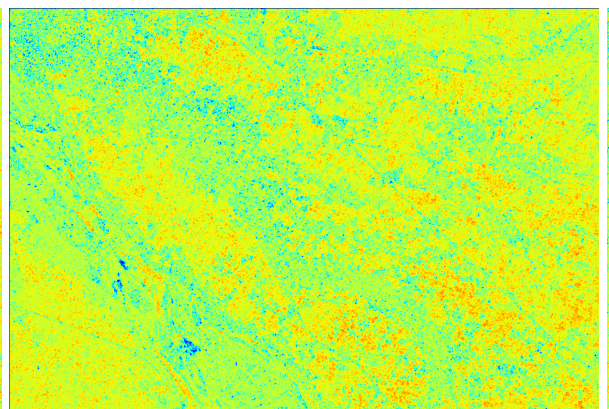

(e) $02-\mathrm{Nov}$

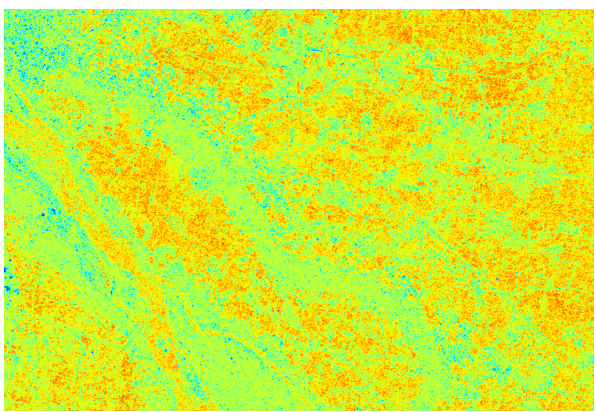

(c) 22-Aug

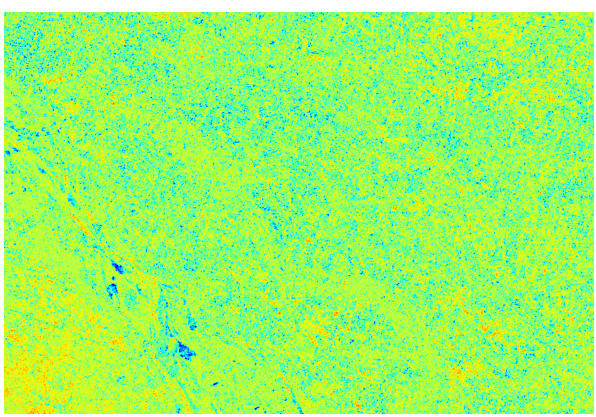

(f) $26-\mathrm{Nov}$

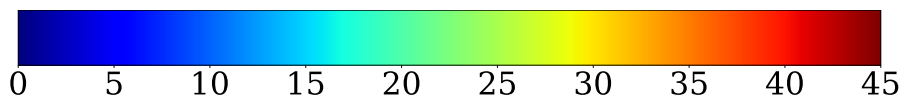

Fig. 14. Time series spatial variation of $\mathcal{C}^{(3)}$ over different rice phenological stages in Vijayawada, India.

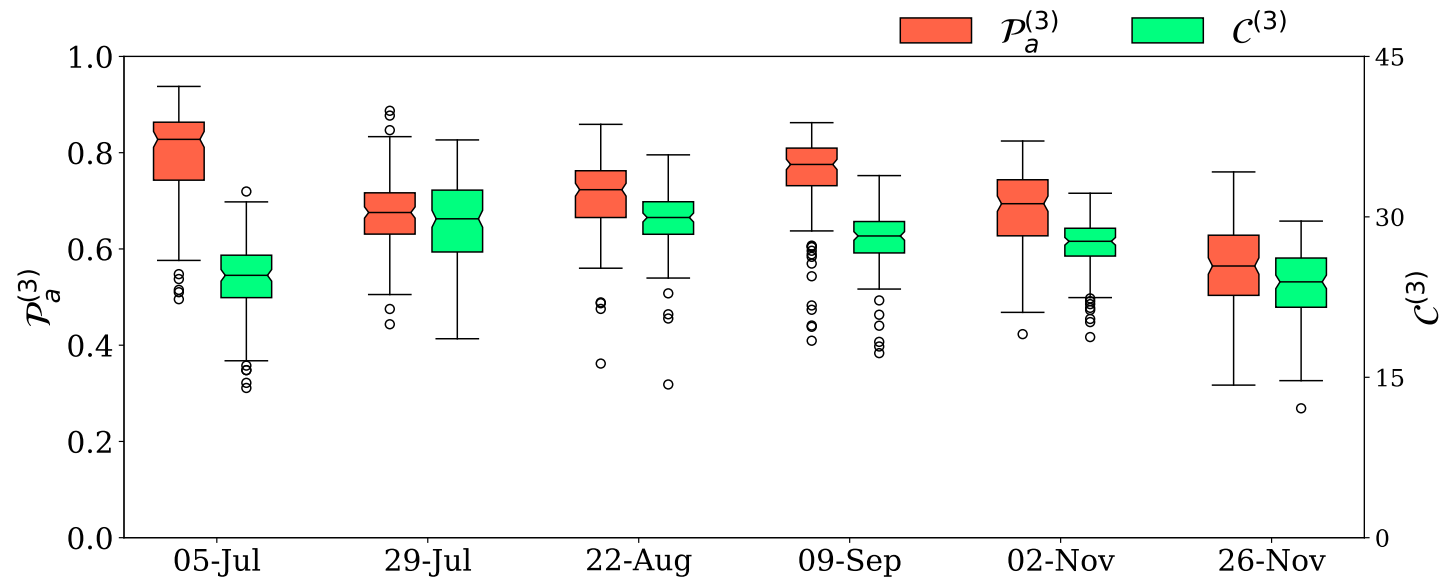

Fig. 15. Temporal variation of $\mathcal{P}_{a}^{3}$ and $\mathcal{C}^{(3)}$ over different rice phenological stages in Vijayawada, India.

of $\mathcal{P}_{a}^{(3)}$ and $\mathcal{C}^{(3)}$ is shown in Fig. 13 and Fig. 14 , respectively.

\section{CONCLUSION}

In this work, we have proposed two rotation invariant parameters: polarimetric purity and complexity to assess the nature of scattering that characterizes (1) polarization structure and (2) scattering mixture, respectively, within a full polarimetric SAR image resolution. In order to express these two measures, we first proposed an alternate expression for the general degree of polarimetric purity. We then formulated the polarimetric purity parameter using the inequalities relating to the bounds of the matrix condition number. It is noteworthy that for the 2D wave polarimetry case, the proposed polarimetric purity is equal to the conventional degree of polarization, hence the 2D Barakat degree of polarization. However, it differs from the Barakat degree of polarization for the 3D case (or higher dimensions). Therefore, the proposed polarimetric purity parameter is more suitable to characterize scattered waves from full polarimetric SAR data.

We proposed a measure of polarimetric complexity which characterizes the mixture of orthogonal canonical targets. Like the purity parameter, we formulated the polarimetric complex- 
ity parameter using the inequalities relating to the bounds of the matrix condition number and the degree of polarimetric purity for equiprobable contributions of orthogonally polarized pure components. At first, we illustrated the variations of the purity and the complexity parameters using two examples: (i) multiple scattering, and (ii) a mixture of canonical targets. The two parameters help characterize targets from polarimetric SAR data. In the multiple scattering case, the proposed purity parameter behaves similarly to the degree of polarimetric purity, i.e., decreasing monotonically with increasing scattering order.

The complexity parameter assists in explaining the mixture among different scattering mechanisms from a target depending on its morphology. We observed significant differences in scattering complexity values between the ocean and urban areas, although the scattering purity is high in both cases. This observation suggests that both are close to being pure scatterers and therefore cannot be well discriminated against using only the purity parameter. In contrast, the complexity parameter reveals that the ocean surface has only one dominant scattering mechanism, while the urban area has one dominant along with a particular second dominant scattering mechanism. Hence, the complexity parameter helps in discriminating scattering characteristics of targets with similar scattering purity information. Additionally, it also helps to identify and understand the mixing of scattering mechanisms at different phenological stages of rice.

One could extend the work by suitably utilizing the $\left(\mathcal{C}^{(3)} / \mathcal{P}_{a}^{(3)}\right)$ plane to characterize time-varying scattering signatures. The two parameters could benefit environmental monitoring using future sensors with frequent revisit and full polarimetric capabilities. It will be worth analyzing the two proposed parameters for high dimensional covariance data (i.e., bistatic, multistatic, etc.) obtained with future SAR sensors and present and future planetary missions.

\section{REPRODUCIBILITY AND REPLICABILITY}

Following the guidelines presented in Ref. [45], we made available the code that supports the reproducibility and replicability of this work in a Github repository: https://github.com/ Subho07/scattering-purity-and-complexity.

\section{APPENDIX}

Barakat [11,17] proposed the $N$-fold measure of his degree of polarization (also referred them as $N D$ polarization measure) as

$$
P_{n}(N)=\left(1-\frac{N^{n} n ! p_{n}(N)}{N^{(n)} p_{1}^{n}(N)}\right)^{1 / 2} ; n=2, \ldots, N
$$

where $N^{(n)}=N(N-1) \cdots(N-n+1)$ with $0 \leq P_{n} \leq 1$ and

$$
\begin{gathered}
p_{1}(N)=\sum \lambda_{1}(N)=\operatorname{tr}\left(\Phi_{N}\right) \\
p_{2}(N)=\sum \lambda_{1}(N) \lambda_{2}(N) \\
\vdots \\
p_{N}(N)=\lambda_{1}(N) \lambda_{2}(N) \cdots \lambda_{N}(N)=\operatorname{det}\left(\Phi_{N}\right) .
\end{gathered}
$$

For $N=3$, there are two corresponding polarization measures of $\Phi_{3}$ as,

$$
\begin{gathered}
P_{3}(3)=\left[1-\frac{27 p_{3}(3)}{p_{1}^{3}(3)}\right]^{1 / 2}=\left[1-\frac{27 \operatorname{det}\left(\Phi_{3}\right)}{\operatorname{tr}^{3}\left(\Phi_{3}\right)}\right]^{1 / 2}, \\
P_{2}(3)=\left[1-\frac{3 p_{2}(3)}{p_{1}^{2}(3)}\right]^{1 / 2} .
\end{gathered}
$$

One can note that equation (21) is the so-called 3D Barakat degree of polarization, whereas equation (22) represents the distance between the unpolarized state and any mixed state. Moreover, for the $2 \times 2$ covariance matrix, equation 16 reduces to the conventional degree of polarization (i.e., wave anisotropy) of a plane wave which one can also express in terms of the four Stokes parameters [15, 16].

\section{ACKNOWLEDGEMENT}

The authors would like to thank Prof. José Jorge Gil Pérez, University of Zaragoza, Spain, for some excellent discussion that has acquainted us with several exciting advances in the field of polarimetry. The authors are highly indebted to Prof. Osvaldo Anibal Rosso, who introduced us to the concept of "complexity" through his phenomenal work in the field of Statistical Complexity Measure (SCM). The authors would like to thank the Canadian Space Agency and MAXAR Technologies Ltd. (formerly MDA) for providing Radarsat2 images through the Joint Experiment for Crop Assessment and Monitoring (JECAM) SAR Inter-comparison Experiment network.

\section{REFERENCES}

[1] S. R. Cloude and E. Pottier, "A review of target decomposition theorems in radar polarimetry," IEEE Trans. Geosci. Remote Sens., vol. 34, no. 2, pp. 498-518, 1996.

[2] — , "An entropy based classification scheme for land applications of polarimetric SAR,” IEEE Trans. Geosci. Remote Sens., vol. 35, no. 1, pp. 68-78, 1997.

[3] J.-S. Lee and E. Pottier, Polarimetric radar imaging: from basics to applications. CRC press, 2009.

[4] R. Touzi, "Target scattering decomposition in terms of roll-invariant target parameters," IEEE Trans. Geosci. Remote Sens., vol. 45, no. 1, pp. 73-84, 2006.

[5] R. Touzi, A. Deschamps, and G. Rother, "Phase of target scattering for wetland characterization using polarimetric c-band SAR," IEEE Transactions on Geoscience and Remote Sensing, vol. 47, no. 9, pp. 3241-3261, 2009.

[6] A. Bhattacharya and R. Touzi, "Polarimetric SAR urban classification using the touzi target scattering decomposition," Canadian Journal of Remote Sensing, vol. 37, no. 4, pp. 323-332, 2011.

[7] K. Li, B. Brisco, S. Yun, and R. Touzi, "Polarimetric decomposition with RADARSAT-2 for rice mapping and monitoring," Canadian Journal of Remote Sensing, vol. 38, no. 2, pp. 169-179, 2012.

[8] D. Ratha, E. Pottier, A. Bhattacharya, and A. C. Frery, "A polsar scattering power factorization framework and novel roll-invariant parameterbased unsupervised classification scheme using a geodesic distance," IEEE Transactions on Geoscience and Remote Sensing, vol. 58, no. 5, pp. 3509-3525, 2019.

[9] S. Dey, A. Bhattacharya, D. Ratha, D. Mandal, and A. C. Frery, "Target characterization and scattering power decomposition for full and compact polarimetric SAR data," IEEE Transactions on Geoscience and Remote Sensing, vol. 59, no. 5, pp. 3981-3998, 2020.

[10] S. Dey, A. Bhattacharya, A. C. Frery, C. López-Martínez, and Y. S. Rao, "A model-free four component scattering power decomposition for polarimetric SAR data," IEEE Journal of Selected Topics in Applied Earth Observations and Remote Sensing, vol. 14, pp. 3887-3902, 2021. 
[11] R. Barakat, "n-fold polarization measures and associated thermodynamic entropy of N partially coherent pencils of radiation," Opt. Acta, vol. 30, no. 8, pp. 1171-1182, 1983.

[12] J. R. Huynen, "Phenomenological theory of radar targets," PhD dissertation, Technical Univ., Delf, The Netherlands, 1970.

[13] S. Dey, A. Bhattacharya, D. Ratha, D. Mandal, H. McNairn, J. M. LopezSanchez, and Y. Rao, "Novel clustering schemes for full and compact polarimetric SAR data: An application for rice phenology characterization," ISPRS Journal of Photogrammetry and Remote Sensing, vol. 169 , pp. $135-151,2020$.

[14] S. Dey, N. Bhogapurapu, A. Bhattacharya, D. Mandal, J. M. LopezSanchez, H. McNairn, and A. C. Frery, "Rice phenology mapping using novel target characterization parameters from polarimetric SAR data," International Journal of Remote Sensing, vol. 42, no. 14, pp. 5519-5543, 2021.

[15] E. Wolf, "Coherence properties of partially polarized electromagnetic radiation," Il Nuovo Cimento (1955-1965), vol. 13, no. 6, pp. 11651181,1959

[16] M. Born and E. Wolf, Principles of optics: electromagnetic theory of propagation, interference and diffraction of light. Elsevier, 2013.

[17] R. Barakat, "Degree of polarization and the principal idempotents of the coherency matrix," Opt. Commun., vol. 23, no. 2, pp. 147-150, 1977.

[18] J. C. Samson, "Descriptions of the Polarization States of Vector Processes: Applications to ULF Magnetic Fields," Geophysical Journal International, vol. 34, no. 4, pp. 403-419, 12 1973. [Online]. Available: https://doi.org/10.1111/j.1365-246X.1973.tb02404.x

[19] C. Brosseau, Fundamentals of polarized light: a statistical optics approach. Wiley-Interscience, 1998.

[20] T. Carozzi, R. Karlsson, and J. Bergman, "Parameters characterizing electromagnetic wave polarization," Phys. Rev. E, vol. 61, pp. 2024 2028, Feb 2000. [Online]. Available: https://link.aps.org/doi/10.1103/ PhysRevE.61.2024

[21] A. Luis, "Degree of polarization for three-dimensional fields as a distance between correlation matrices," Optics Communications, vol. 253, no. 1, pp. 10-14, 2005. [Online]. Available: https: //www.sciencedirect.com/science/article/pii/S0030401805003937

[22] J. J. G. Pérez and R. Ossikovski, Polarized light and the Mueller matrix approach. CRC press, 2017.

[23] J. J. Gil, "Polarimetric characterization of light and media: Physical quantities involved in polarimetric phenomena," The European Physical Journal - Applied Physics, vol. 40, no. 1, p. 1-47, 2007.

[24] I. San José and J. J. Gil, "Invariant indices of polarimetric purity: generalized indices of purity for $\mathrm{n} \times \mathrm{n}$ covariance matrices," Optics communications, vol. 284, no. 1, pp. 38-47, 2011.

[25] C. Marechal, E. Pottier, L. Hubert-Moy, and S. Rapinel, "One year wetland survey investigations from quad-pol radarsat-2 time-series SAR images," Canadian Journal of Remote Sensing, vol. 38, no. 3, pp. 240-252, 2012. [Online]. Available: https://doi.org/10.5589/m12-017

[26] R. Shirvany, M. Chabert, and J.-Y. Tourneret, "Estimation of the degree of polarization for hybrid/compact and linear dual-pol SAR intensity images: Principles and applications," IEEE transactions on geoscience and remote sensing, vol. 51, no. 1, pp. 539-551, 2012.

[27] — "Ship and oil-spill detection using the degree of polarization in linear and hybrid/compact dual-pol SAR," IEEE Journal of Selected Topics in Applied Earth Observations and Remote Sensing, vol. 5, no. 3, pp. 885-892, 2012.

[28] M. Mahdianpari, F. Mohammadimanesh, H. McNairn, A. Davidson, M. Rezaee, B. Salehi, and S. Homayouni, "Mid-season crop classification using dual-, compact-, and full-polarization in preparation for the radarsat constellation mission ( $\mathrm{rcm})$," Remote Sensing, vol. 11, no. 13, 2019. [Online]. Available: https://www.mdpi.com/2072-4292/ $11 / 13 / 1582$

[29] D. P. Feldman and J. P. Crutchfield, "Measures of statistical complexity: Why?" Physics Letters A, vol. 238, no. 4-5, pp. 244-252, 1998. [Online]. Available: http://www.sciencedirect.com/science/article/ pii/S0375960197008554

[30] S. Cloude, Polarisation: applications in remote sensing. OUP Oxford, 2009.

[31] H. Wolkowicz and G. P. H. Styan, "Bounds for eigenvalues using traces," Linear Algebra and Its Applications, vol. 29, pp. 471-506, 1980.

[32] J. J. Van Zyl, H. A. Zebker, and C. Elachi, "Imaging radar polarization signatures: Theory and observation," Radio science, vol. 22, no. 04, pp. 529-543, 1987.

[33] H. A. Zebker, J. J. van Zyl, and D. N. Held, "Imaging radar polarimetry from wave synthesis," Journal of Geophysical Research: Solid Earth, vol. 92, no. B1, pp. 683-701, 1987. [Online]. Available: https: //agupubs.onlinelibrary.wiley.com/doi/abs/10.1029/JB092iB01p00683
[34] M. Martin, A. Plastino, and O. Rosso, "Generalized statistical complexity measures: Geometrical and analytical properties," Physica A: Statistical Mechanics and its Applications, vol. 369, no. 2, pp. 439-462, 2006. [Online]. Available: https://www.sciencedirect.com/ science/article/pii/S0378437106001324

[35] O. A. Rosso, L. De Micco, H. A. Larrondo, M. T. Martín, and A. Plastino, "Generalized statistical complexity measure," International Journal of Bifurcation and Chaos, vol. 20, no. 03, pp. 775-785, 2010. [Online]. Available: https://doi.org/10.1142/S021812741002606X

[36] R. López-Ruiz, H. Mancini, and X. Calbet, "A statistical measure of complexity," Physics Letters A, vol. 209, no. 5, pp. 321-326, 1995. [Online]. Available: https://www.sciencedirect.com/science/article/pii/ 0375960195008675

[37] J. J. Van Zyl, Synthetic aperture radar polarimetry. John Wiley \& Sons, 2011, vol. 2.

[38] D. Ratha, E. Pottier, A. Bhattacharya, and A. C. Frery, "A polsar scattering power factorization framework and novel roll-invariant parameterbased unsupervised classification scheme using a geodesic distance," IEEE Trans. Geosci. Remote Sens., pp. 1-17, 2019

[39] A. Freeman and S. L. Durden, "A three-component scattering model for polarimetric SAR data," IEEE Trans. Geosci. Remote Sens., vol. 36 , no. 3, pp. 963-973, 1998

[40] Dominique Bicout and Christian Brosseau, "Multiply scattered waves through a spatially random medium : entropy production and depolarization," J. Phys. I France, vol. 2, no. 11, pp. 2047-2063, 1992. [Online]. Available: https://doi.org/10.1051/jp1:1992266

[41] J.-S. Lee and E. Pottier, Polarimetric radar imaging: from basics to applications. CRC press, 2017.

[42] Y. Yamaguchi, A. Sato, W.-M. Boerner, R. Sato, and H. Yamada, "Fourcomponent scattering power decomposition with rotation of coherency matrix," IEEE Trans. Geosci. Remote Sens., vol. 49, no. 6, pp. 22512258, 2011.

[43] R. Guinvarc'h and L. Thirion-Lefevre, "Cross-polarization amplitudes of obliquely orientated buildings with application to urban areas," IEEE Geosci. Remote Sens. Lett., vol. 14, no. 11, pp. 1913-1917, 2017.

[44] Z. He, S. Li, Y. Wang, L. Dai, and S. Lin, "Monitoring rice phenology based on backscattering characteristics of multi-temporal RADARSAT-2 datasets," Remote Sensing, vol. 10, no. 2, p. 340, 2018

[45] A. C. Frery, L. Gomez, and A. C. Medeiros, "A badging system for reproducibility and replicability in remote sensing research," IEEE $J$. Sel. Topics Appl. Earth Observ. Remote Sens., vol. 13, pp. 4988-4995, 2020.

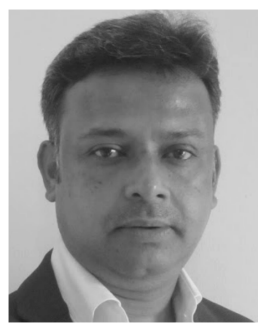

Avik Bhattacharya (M'08-SM'16) received the integrated M.Sc. degree in Mathematics from the Indian Institute of Technology, Kharagpur, India, in 2000 and the Ph.D. degree in remote sensing image processing and analysis from Télécom ParisTech, Paris, France, and the Ariana Research Group, Institut National de Recherche en Informatique et en Automatique (INRIA), Sophia Antipolis, Nice, France, in 2007. He is currently an Associate Professor at the Centre of Studies in Resources Engineering, Indian Institute of Technology Bombay (CSRE, IITB), Mumbai, India. Before joining IITB, he was a Canadian Government Research Fellow at the Canadian Centre for Remote Sensing (CCRS) in Ottawa, ON, Canada. He received the Natural Sciences and Engineering Research Council of Canada visiting scientist fellowship at the Canadian national laboratories, from 2008 to 2011. His current research interests include SAR polarimetry, statistical analysis of polarimetric SAR images, applications of Radar Remote Sensing in Agriculture, Cryosphere, Urban and Planetary studies. Dr. Bhattacharya is the Editor-in-Chief of IEEE Geoscience and Remote Sensing Letters. He was an Associate Editor of IEEE GRSL. He has been the Guest Editor of the special issue on Applied Earth Observations and Remote Sensing in India in IEEE Journal of Selected Topics in Applied Earth Observations and Remote Sensing (J-STARS), 2017. He was one of the guest editors of the special stream on Advanced Statistical Techniques in SAR Image Processing and Analysis in IEEE Geoscience and Remote Sensing LetterS, 2018. He is the Founding Chairperson of the IEEE Geoscience and Remote Sensing Society (GRSS) Chapter of the Bombay Section. He is currently leading the Microwave Remote Sensing Lab (www.mrslab.in) at CSRE, IITB. 


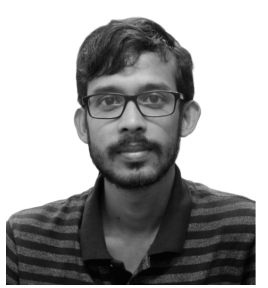

Subhadip Dey (S'17) received the B.Tech degree in Agricultural Engineering from Bidhan Chandra Krishi Viswavidyalaya, India. He received the M. Tech degree in Aquacultural Engineering, Department of Agricultural and Food Engineering, Indian Institute of Technology Kharagpur, India. He is currently pursuing the Ph.D. degree at the Microwave Remote Sensing Lab, Centre of Studies in Resources Engineering (CSRE), Indian Institute of Technology Bombay, India. His current research interests are land cover classification and agricultural crop mapping and monitoring using Synthetic Aperture Radar data.

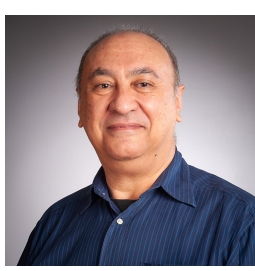

Alejandro C. Frery (S'92-SM'03) received the B.Sc. degree in Electronic and Electrical Engineering from the Universidad de Mendoza, Mendoza, Argentina. His M.Sc. degree was in Applied Mathematics (Statistics) from the Instituto de Matemática Pura e Aplicada (IMPA, Rio de Janeiro) and his Ph.D. degree was in Applied Computing from the Instituto Nacional de Pesquisas Espaciais (INPE, São José dos Campos, Brazil). He was the founder of LaCCAN - Laboratório de Computação Científica e Análise Numérica, Universidade Federal de Alagoas, Maceió, Brazil, and holds a Huashan Scholar position (2019-2021) with the Key Lab of Intelligent Perception and Image Understanding of the Ministry of Education, Xidian University, Xi' an, China. He was the Editor-in-Chief of IEEE Geoscience and Remote Sensing Letters (2014-2018). In 2018 he received the IEEE GRSS Regional Leader Award. His research interests are statistical computing and stochastic modeling. 